\title{
Spectral-element simulations of long-term fault slip: Effect of low-rigidity layers on earthquake-cycle dynamics
}

\author{
Y. Kaneko, ${ }^{1}$ J.-P. Ampuero, ${ }^{2}$ and N. Lapusta ${ }^{2,3}$ \\ Received 26 March 2011; revised 1 July 2011; accepted 15 July 2011; published 29 October 2011.
}

[1] We develop a spectral element method for the simulation of long-term histories of spontaneous seismic and aseismic slip on faults subjected to tectonic loading. Our approach reproduces all stages of earthquake cycles: nucleation and propagation of earthquake rupture, postseismic slip and interseismic creep. We apply the developed methodology to study the effects of low-rigidity layers on the dynamics of the earthquake cycle in 2-D. We consider two cases: small $(\mathrm{M} \sim 1)$ earthquakes on a fault surrounded by a damaged fault zone and large $(\mathrm{M} \sim 7)$ earthquakes on a vertical strike-slip fault that cuts through shallow low-rigidity layers. Our results indicate how the source properties of repeating earthquakes are affected by the presence of a damaged fault zone with low rigidity. Compared to faults in homogeneous media, we find (1) reduction in the earthquake nucleation size, (2) amplification of slip rates during dynamic rupture propagation, (3) larger recurrence interval, and (4) smaller amount of aseismic slip. Based on linear stability analysis, we derive a theoretical estimate of the nucleation size as a function of the width and rigidity reduction of the fault zone layer, which is in good agreement with simulated nucleation sizes. We further examine the effects of vertically-stratified layers (e.g., sedimentary basins) on the nature of shallow coseismic slip deficit. Our results suggest that low-rigidity shallow layers alone do not lead to coseismic slip deficit. While the low-rigidity layers result in lower interseismic stress accumulation, they also cause dynamic amplification of slip rates, with the net effect on slip being nearly zero.

Citation: Kaneko, Y., J.-P. Ampuero, and N. Lapusta (2011), Spectral-element simulations of long-term fault slip: Effect of low-rigidity layers on earthquake-cycle dynamics, J. Geophys. Res., 116, B10313, doi:10.1029/2011JB008395.

\section{Introduction}

[2] Earthquake cycle simulations are important for understanding earthquake mechanics and physics-based hazard analysis. Modeling long-term slip histories of faults remains, however, quite challenging due to a wide range of spatial and temporal scales involved. To simulate a spontaneous earthquake sequence on a fault, a model needs to incorporate and resolve slow tectonic loading during the interseismic periods, nucleation and propagation of rupture during earthquakes that involve rapid changes in stress and slip rate at the propagating dynamic rupture tips, and the subsequent postseismic deformation and aseismic afterslip.

\footnotetext{
${ }^{1}$ Institute of Geophysics and Planetary Physics, Scripps Institution of Oceanography, University of California, San Diego, La Jolla, California, USA.

${ }^{2}$ Division of Geological and Planetary Sciences, California Institute of Technology, Pasadena, California, USA.

${ }^{3}$ Division of Engineering and Applied Science, California Institute of Technology, Pasadena, California, USA.

Copyright 2011 by the American Geophysical Union. 0148-0227/11/2011JB008395
}

In addition, destructive large earthquakes occur on faults that extend tens to hundreds of kilometers while variations in stress changes and slip rate at the rupture tip occur over distances of the order of meters.

[3] Many approaches to modeling long-term histories of fault slip have been proposed [e.g., Shibazaki and Matsu'ura, 1992; Rice, 1993; Cochard and Madariaga, 1996; Tullis, 1996; Ward, 1997; Rundle et al., 1999; Kato, 2004; Duan and Oglesby, 2005; Liu and Rice, 2005; Hillers et al., 2006; Dieterich and Richards-Dinger, 2010] but all of them used simplified treatments of either aseismic slip processes (e.g., nucleation, fault creep, and afterslip) or inertial effects during dynamic rupture. Lapusta et al. [2000] and Lapusta and Liu [2009] developed 2-D and 3-D boundary integral methods (BIMs) capable of capturing both seismic and aseismic slip and the gradual process of earthquake nucleation. Those studies are restricted to planar faults embedded in a uniform elastic space or a half-space. At the same time, observations indicate complex crustal structures with variable bulk properties, fault damage zones, and non-planar fault geometries. Hence it is important to include those factors into long-term earthquake cycle models, combining them with laboratoryderived fault constitutive relations. 
[4] In this work, we develop a spectral element method (SEM) that can enable us to simulate long-term history of spontaneous seismic and aseismic slip on a fault embedded into heterogeneous bulk and subjected to laboratory-derived rate and state friction and slow tectonic loading. In particular, we present a quasi-static SEM with a time updating scheme that can be used to model long-term deformation histories and that is suitable for a fault boundary governed by a rate and state friction formulation. Our model merges the explicit scheme presented by Kaneko et al. [2008] for simulating dynamic rupture propagation with the quasi-static SEM developed in this work for modeling slow tectonic loading and the associated crustal deformation and fault slip. The SEM with an explicit time scheme was built upon prior studies by Komatitsch and Vilotte [1998], Komatitsch and Tromp [1999], and Ampuero [2002]. The combined algorithm is able to resolve all stages of an earthquake cycle, including gradual nucleation processes, dynamic rupture propagation, postseismic slip, and aseismic processes throughout the loading period. Since the presented approach is based on finite element methodology, it can be adapted to simulations of non-planar faults and fault systems.

[5] Our methodology is described in sections 2 and 3. Section 3 also illustrates its potential by presenting simulations of long-term slip on a fault segment with relatively simple distributions of fault friction properties. To verify the developed SEM approach, we compare SEM and BIM simulation results in a 2-D model of small $(\mathrm{M} \sim 1)$ repeating earthquakes. We then consider two application examples to explore the potential effects of variable bulk properties on repeating earthquakes (sections 4 and 5).

[6] In section 4, we consider small repeating earthquakes on a fault that bisects a meter-scale fault-parallel low-rigidity zone embedded in undamaged or damaged but higherrigidity host rock. Such a model configuration is motivated by localized damaged zones surrounding fault cores often found on exhumed faults [e.g., Chester et al., 1993]. Recently, meter-thick foliated fault gouge of extremely low $P$ - and $S$-wave speeds embedded within a $200-\mathrm{m}$ wide damaged zone at 2.6-2.8 km depths was found in the EarthScope's San Andreas Fault Observatory at Depth (SAFOD) project [Zoback et al., 2010]. Simulations of single earthquake rupture suggest that such a mechanical configuration leads to perturbed rupture speeds and slip velocity of propagating rupture, resulting in high-frequency oscillations in the slip function near the rupture front [Harris and Day, 1997]. Here we examine additional effects of a fault-parallel low-rigidity zone on earthquake source properties of simulated repeating earthquakes. We compare the model response in a layered bulk with that in a homogeneous bulk, and investigate how earthquake source properties, such as stress drop, recurrence intervals, and nucleation sizes, depend on the width of a low-rigidity layer. Given the recent successes of rate and state models in explaining several properties of repeating earthquake sequences [Chen and Lapusta, 2009; Chen et al., 2010], it is important to consider these additional effects for proper interpretation of observations.

[7] In section 5, we consider the effects of stratified bulk properties (e.g., a sedimentary basin) on simple repeating earthquakes that rupture the entire seismogenic depth. Fialko et al. [2005] pointed out that, for several large (M 7) strike-slip earthquakes, coseismic slip in the uppermost crust is systematically lower than that at seismogenic depth. A reduction of coseismic slip at shallow depths $(<3-4 \mathrm{~km})$, referred to as 'shallow slip deficit', has been inferred for several large strike-slip crustal earthquakes [e.g., Simons et al., 2002; Fialko et al., 2005; Bilham, 2010], including the 1992 M7.3 Landers earthquake, the 1999 M7.1 Hector Mine earthquake, the 2005 M6.5 Bam earthquake, and the 2010 M7.0 Haiti earthquake. Several mechanisms have been proposed to explain the shallow slip deficit, including the presence of velocity-strengthening friction at shallow depths that releases accumulated strain by fault creep [e.g., Marone et al., 1991; Marone, 1998; Kaneko et al., 2008] and low pre-stress in low-rigidity shallow bulk materials resulting from uniform tectonic strain [e.g., Rybicki, 1992; Rybicki and Yamashita, 1998]. Using the developed SEM, we investigate whether a vertical strike-slip fault embedded in a vertically stratified bulk structure can cause shallow coseismic slip deficit without the presence of a shallow velocity-strengthening region.

\section{A Quasi-Static SEM Algorithm for Simulations of Long-Term Deformation Histories}

\subsection{Discretized Elastodynamic Relations}

[8] The SEM dynamic model presented by Kaneko et al. [2008] relies on an explicit time updating scheme, the approach commonly used in SEMs for wave propagation [e.g., Komatitsch and Vilotte, 1998; Komatitsch and Tromp, 1999]. However, the explicit time scheme limits the maximum length of each time step $\Delta t$ by the Courant stability condition. For dynamic rupture simulations, the Courant condition can be rewritten in terms of the cohesive zone size $\Lambda$ divided by the wave speeds of the medium:

$$
\Delta t \leq \frac{C \Lambda}{\sqrt{D} N V_{\mathrm{p} \mathrm{or} \mathrm{s}}},
$$

where $D$ is the dimension of the problem, $C$ is a stability parameter that depends only on the time scheme and is of order one, and $N$ is the number of fault plane node points within the cohesive zone. (In SEM, the critical time step is actually smaller due to the non-uniform distribution of the Gauss-Lobatto-Legendre nodes inside each spectral element.) $N$ should be at least 3-5 for well-resolved simulations of dynamic rupture in the cases with slip-weakening or weakly rate-dependent friction laws [Day et al., 2005; Kaneko et al., 2008; Kaneko and Lapusta, 2010]. For dynamic rupture simulations with cohesive zone sizes of 1-100 meters, $\Delta t \sim$ $10^{-4}-10^{-2} \mathrm{~s}$, and hence simulating tens to thousands of years of deformation histories is not computationally feasible. To take a longer time step, one needs to use an implicit time updating scheme. SEMs with implicit schemes have been used to solve elastic and acoustic wave equations [e.g., Ampuero, 2002; Zampieri and Pavarino, 2006; Dupros et al., 2010]. Here we develop a quasi-static SEM with an adaptive time stepping and merge it with the fully dynamic SEM to simulate long-term slip histories on a rate-and-state fault.

[9] As in the work of Kaneko et al. [2008], we start from the discretized weak form of the equation of motion in its matrix form:

$$
\mathbf{M} \ddot{\mathbf{u}}=-\mathbf{K u}+\mathbf{B} \boldsymbol{\tau}
$$




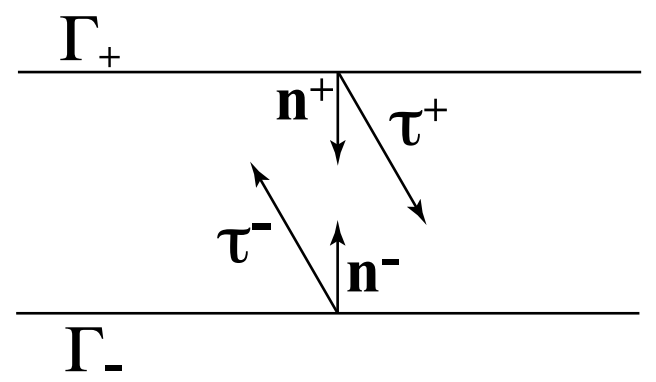

Figure 1. The fault divided into two non-overlapping surfaces $\Gamma_{ \pm}$.

where $\mathbf{M}$ and $\mathbf{K}$ are the mass and stiffness matrix respectively, $\mathbf{B}$ is the fault-boundary matrix (described in Appendix A), $\boldsymbol{\tau}=\boldsymbol{\tau}^{\text {tot }}-\boldsymbol{\tau}_{\mathrm{o}}$ is the relative traction vector on the fault, $\tau^{\text {tot }}$ is the total traction and $\tau_{\mathrm{o}}$ is the traction in the reference static-equilibrium state. Vectors $\mathbf{u}, \dot{\mathbf{u}}$, and $\ddot{\mathbf{u}}$ collect the values of displacements, particle velocities and accelerations, respectively, of all the computational nodes of the bulk mesh.

[10] In the case of quasi-static problems, equation (2) becomes

$$
\mathbf{K u}=\mathbf{B} \tau .
$$

Let us decompose the displacement vector $\mathbf{u}$ into the values on fault nodes, denoted by $\mathbf{u}^{\mathrm{f}}$, and the values on nodes within the medium, denoted by $\mathbf{u}^{\mathrm{m}}$. Then

$$
\begin{gathered}
\mathbf{K}_{11} \mathbf{u}^{\mathrm{f}}+\mathbf{K}_{12} \mathbf{u}^{\mathrm{m}}=\mathbf{B} \boldsymbol{\tau}, \\
\mathbf{K}_{21} \mathbf{u}^{\mathrm{f}}+\mathbf{K}_{22} \mathbf{u}^{\mathrm{m}}=\mathbf{0},
\end{gathered}
$$

where $\mathbf{K}_{11}$ and $\mathbf{K}_{12}$ are the parts of the stiffness matrix corresponding to $\mathbf{u}^{\mathrm{f}}$, and $\mathbf{K}_{21}$ and $\mathbf{K}_{22}$ are the parts corresponding to $\mathbf{u}^{\mathrm{m}}$. From equation (5), we have

$$
\mathbf{K}_{22} \mathbf{u}^{\mathrm{m}}=-\mathbf{K}_{21} \mathbf{u}^{\mathrm{f}} .
$$

Given the displacement on the fault, $\mathbf{u}^{\mathrm{f}}$, this equation yields the corresponding displacement field in the medium, $\mathbf{u}^{\mathrm{m}}$.

[11] In equation (4), let us introduce $\mathbf{f} \equiv \mathbf{K}_{11} \mathbf{u}^{\mathrm{f}}+\mathbf{K}_{12} \mathbf{u}^{\mathrm{m}}$. We now write equation (4) for the fault nodes with the \pm signs indicating the values of field variables on the two sides of the fault (Figure 1):

$$
\mathbf{B}_{ \pm} \boldsymbol{\tau}_{ \pm}=\mathbf{f}_{ \pm}
$$

Subtracting the minus side from the plus side, and using the sign convention $\boldsymbol{\tau}=-\boldsymbol{\tau}_{+}=\boldsymbol{\tau}_{-}$, where $\boldsymbol{\tau}_{ \pm}$are defined with respect to the outward normal from the fault boundary $\Gamma_{ \pm}$ (Figure 1), we obtain

$$
\boldsymbol{\tau}=-\left(\mathbf{B}_{+}+\mathbf{B}_{-}\right)^{-1}\left(\mathbf{f}_{+}-\mathbf{f}_{-}\right) .
$$

Equation (6) allows to eliminate the off-fault degrees of freedom, $\mathbf{u}^{\mathrm{m}}$, to obtain a formulation (8) involving only the degrees of freedom on the fault $\left(\boldsymbol{\tau}\right.$ and $\left.\mathbf{u}^{\mathrm{f}}\right)$. This procedure is known as static condensation or sub-structuring in computational mechanics. Because the $\mathbf{B}_{ \pm}$matrices are diagonal, the expression (8) is a local relation which can be computed node by node on the fault once the $\mathbf{f}_{ \pm}$terms are known or predictor values are assumed. It is convenient to rewrite equation (8) in terms of total traction, $\tau^{\text {tot }}=\tau_{\mathrm{o}}+\tau$ :

$$
\tau^{\mathrm{tot}}=\tau_{\mathrm{o}}+\boldsymbol{\tau}=\boldsymbol{\tau}_{\mathrm{o}}-\left(\mathbf{B}_{+}+\mathbf{B}_{-}\right)^{-1}\left(\mathbf{f}_{+}-\mathbf{f}_{-}\right) .
$$

Note that for the cases we consider in this study, the faultnormal components of traction $\tau$ remain unchanged, and hence the fault-normal components of $\tau$ are neglected in the calculations.

[12] The developed quasi-static time stepping algorithm is summarized in Appendix B. The algorithm is written in general terms, independent of specific forms of fault constitutive relations. The quasi-static algorithm is similar to Heun's method, which can be seen as an extension of the Euler method into a two-stage second-order Runge-Kutta method. While Heun's method is usually qualified as an explicit method, we still need to solve a large linear system during static condensation. Hence we prefer to qualify our algorithm as implicit. In section 2.3 , we discuss the quasistatic time updating scheme coupled with a specific form of friction laws in more detail.

\subsection{Fault Constitutive Response: Rate and State Friction Laws}

[13] The fault resistance to sliding is described by laboratory-derived rate and state friction laws, which were developed to incorporate observations of rock friction experiments at low slip rate [Dieterich, 1978, 1979; Ruina, 1983; Blanpied et al., 1995, 1998; Marone, 1998]. For timeindependent effective normal stress $\bar{\sigma}$, the shear strength $\mathcal{T}$ on the fault is expressed as

$$
\begin{aligned}
\mathcal{T} & =\psi(\dot{\delta}, \theta) \\
& =\bar{\sigma}\left[f_{\mathrm{o}}+a \ln \left(\frac{\dot{\delta}}{\dot{\delta}_{\mathrm{o}}}\right)+b \ln \left(\frac{\dot{\delta}_{\mathrm{o}} \theta}{L}\right)\right],
\end{aligned}
$$

where $a$ and $b$ are rate and state constitutive parameters with magnitudes of the order of $0.01, \dot{\delta}$ is the magnitude of slip velocity, $f_{0}$ is a reference friction coefficient corresponding to a reference slip velocity $\dot{\delta}_{\mathrm{o}}, \theta$ is a state variable which is typically interpreted as the average age of the population of contacts between two surfaces, and $L$ is the characteristic slip for state evolution [Dieterich, 1978, 1979; Rice and Ruina, 1983; Ruina, 1983; Dieterich and Kilgore, 1994]. Two types of state-variable evolution laws are commonly used in modeling:

$$
\begin{gathered}
\frac{d \theta}{d t}=1-\frac{\dot{\delta} \theta}{L} \quad(\text { aging law }), \\
\left.\frac{d \theta}{d t}=-\frac{\dot{\delta} \theta}{L} \ln \left(\frac{\dot{\delta} \theta}{L}\right) \quad \text { (slip law }\right) .
\end{gathered}
$$

[14] The parameter combination $a-b<0$ corresponds to steady state velocity-weakening friction and can lead to unstable slip, whereas $a-b>0$ corresponds to steady state velocity-strengthening and leads to stable sliding [Rice and 
Ruina, 1983; Ruina, 1983]. Throughout this article, we omit the words "steady state" and simply refer to velocity weakening/strengthening.

[15] In expression (10), shear frictional strength $\mathcal{T}$ is undefined for slip velocities $\dot{\delta}=0$, which is unphysical. To regularize (10) near $\dot{\delta}=0$, we follow the approach of Rice and Ben-Zion [1996] and Lapusta et al. [2000] in using a thermally activated creep model of the direct effect term $a$ $\ln \left(\dot{\delta} / \dot{\delta}_{\mathrm{o}}\right)$ to obtain

$$
\begin{aligned}
\mathcal{T} & =\psi(\dot{\delta}, \theta) \\
& =a \bar{\sigma} \operatorname{arcsinh}\left[\frac{\dot{\delta}}{2 \dot{\delta}_{\mathrm{o}}} \exp \left(\frac{f_{\mathrm{o}}+b \ln \left(\dot{\delta}_{\mathrm{o}} \theta / L\right)}{a}\right)\right] .
\end{aligned}
$$

Solving (13) for $\dot{\delta}$ gives

$$
\begin{aligned}
\dot{\delta} & =\phi(\mathcal{T}, \theta) \\
& =2 \delta_{\mathrm{o}} \sinh \left(\frac{\mathcal{T}}{a \bar{\sigma}}\right) \exp \left(\frac{-f_{\mathrm{o}}-b \ln \left(\dot{\delta}_{\mathrm{o}} \theta / L\right)}{a}\right) .
\end{aligned}
$$

This regularization produces a negligible change from equation (10) in the range of slip velocities explored by laboratory experiments; the difference in $\dot{\delta}$ at $\dot{\delta} \sim \dot{\delta}_{\mathrm{o}}$ is of the order of $\exp \left(-2 f_{0} / a\right)$ or less, and the typical value of $f_{0} / a$ in this study is 40 .

[16] Under slow tectonic loading, frictional instability (i.e., an earthquake) is able to develop only if the velocityweakening region of the fault exceeds the nucleation size $h^{*}$ [Rice and Ruina, 1983; Rice, 1993; Rubin and Ampuero, 2005]. Two theoretical estimates of the earthquake nucleation size for 2-D problems are given by

$$
\begin{aligned}
& h_{\mathrm{RR}}^{*}=\frac{\pi}{4} \frac{\mu^{*} L}{\bar{\sigma}(b-a)}, \\
& h_{\mathrm{RA}}^{*}=\frac{2}{\pi} \frac{\mu^{*} L b}{\bar{\sigma}(b-a)^{2}},
\end{aligned}
$$

where $\mu^{*}=\mu$ for mode III and $\mu^{*}=\mu /(1-\nu)$ for mode II. The estimate $h_{\mathrm{RR}}^{*}$ was derived from the linear stability analysis of steady sliding by Rice and Ruina [1983], while $h_{\mathrm{RA}}^{*}$ was obtained for the parameter regime $a / b>0.5$ by Rubin and Ampuero [2005] on the basis of energy balance for a quasi-statically expanding crack. Note that Rubin and Ampuero [2005] gave formulae for half of the nucleation size but we use full nucleation sizes here.

\subsection{Updating Scheme: Advancing One Evolution Time Step}

[17] We have developed an updating scheme appropriate for the rate and state fault boundary condition. Here, we discuss how values of field variables are updated over one evolution time step. We adopt a multistage predictor-corrector strategy to solve the statically condensed problem. Suppose that the discretized values of displacement $\mathbf{u}$ and particle velocity $\dot{\mathbf{u}}$ are known at the $n$th time step. To find the values of the field variables at the $(n+1)$ th time step, we perform the following steps.
[18] 1. Predict the values of displacements on the fault $\mathbf{u}^{\mathrm{f}}$, based on the known values at the $n$th time step:

$$
\mathbf{u}_{n+1}^{* \mathrm{f}}=\mathbf{u}_{n}^{\mathrm{f}}+\Delta t \dot{\mathbf{u}}_{n}^{\mathrm{f}} .
$$

[19] 2. Solve for the displacement field in the medium $\mathbf{u}_{n+1}^{* m}$ using equation (6):

$$
\mathbf{K}_{22} \mathbf{u}_{n+1}^{* \mathrm{~m}}=-\mathbf{K}_{21} \mathbf{u}_{n+1}^{* \mathrm{f}} .
$$

This is solved by a preconditioned conjugate gradient method, an iterative method for solving symmetric-positivedefinite systems of equations. The algorithm we use is based on Hestenes and Stiefel [1952] and is summarized by Trefethen and Bau [1997]. Because the stiffness matrix $\mathbf{K}_{22}$ is large $\left(\sim 10^{5}\right.$ by $\left.10^{5}\right)$, a direct method such as Gaussian elimination cannot be used. Fortunately, the matrix $\mathbf{K}_{22}$ is sparse, and the product $\mathbf{K u}$ is always computed at a local elemental level as in the case of the dynamic SEM [Kaneko et al., 2008]. This is why we use an iterative method.

[20] 3. Compute $\mathbf{f}^{*}=\mathbf{K}_{11} \mathbf{u}_{n+1}^{* \mathrm{f}}+\mathbf{K}_{12} \mathbf{u}_{n+1}^{* \mathrm{~m}}$ and $\boldsymbol{\tau}_{n+1}^{* \text { tot }}$ in equation (9):

$$
\boldsymbol{\tau}_{n+1}^{\mathrm{tot} *}=\boldsymbol{\tau}_{\mathrm{o}}-\left(\mathbf{B}_{+}+\mathbf{B}_{-}\right)^{-1}\left(\mathbf{f}_{+, n+1}^{*}-\mathbf{f}_{-, n+1}^{*}\right) .
$$

[21] 4. Determine the first prediction of the state variable, $\theta_{n+1}^{*}$. By integrating the evolution law (11) or (12) with the constant magnitude $\dot{\delta}_{n}$ of slip velocity $\dot{\boldsymbol{\delta}}_{n}=\mathbf{u}_{n}^{+}-\mathbf{u}_{n}^{-}$during the time step, we obtain

$$
\theta^{*}{ }_{n+1}=\theta_{n} \exp \left(-\frac{\dot{\delta}_{n} \Delta t}{L}\right)+\frac{L}{\dot{\delta}_{n}}\left(1-\exp \left(-\frac{\dot{\delta}_{n} \Delta t}{L}\right)\right)
$$

for the aging law, and

$$
\theta^{*}{ }_{n+1}=\frac{L}{\dot{\delta}_{n}}\left(\frac{\dot{\delta}_{n} \theta_{n}}{L}\right)^{\exp \left(-\dot{\delta}_{n} \Delta t / L\right)}
$$

for the slip law.

[22] 5. Find the first prediction of slip velocity $\dot{\delta}_{n+1}^{*}$ by equating the magnitude of shear stress in equation (19) and strength in equation (13). The directions of shear traction vector $\tau_{n+1}^{\text {tot }}$ and slip velocity vector $\dot{\boldsymbol{\delta}}_{n+1}$ have to coincide. From equation (19), the traction $\tau_{n+1}^{* \text { tot }}$ and $\mathbf{f}_{n+1}^{*}$ have the same direction. By projecting (19) onto that direction and using equation (14), we obtain

$$
\dot{\delta}_{n+1}^{*}=\phi\left(\tau_{n+1}^{\mathrm{tot} *}, \theta_{n+1}^{*}\right)
$$

Using the directional cosines constructed from the components of $\tau_{n+1}^{* \text { tot }}$, we obtain the components of $\dot{\delta}_{n+1}^{*}$.

[23] 6. Calculate the final prediction of displacement and slip on the fault, $\mathbf{u}_{n+1}^{* * \mathrm{f}}=\frac{1}{2} \boldsymbol{\delta}_{n+1}^{* *}$, at the $(n+1)$ th time step by

$$
\mathbf{u}_{n+1}^{* * \mathrm{f}}=\mathbf{u}_{n}^{\mathrm{f}}+\frac{\Delta t}{2}\left(\dot{\mathbf{u}}_{n}^{\mathrm{f}}+\dot{\mathbf{u}}_{n+1}^{* \mathrm{f}}\right) .
$$

[24] 7. Make the corresponding prediction $\mathbf{u}_{n+1}^{* * m}$ of the displacement in the medium using the $\mathbf{u}_{n+1}^{* * \mathrm{f}}$ as in step 2 . This 

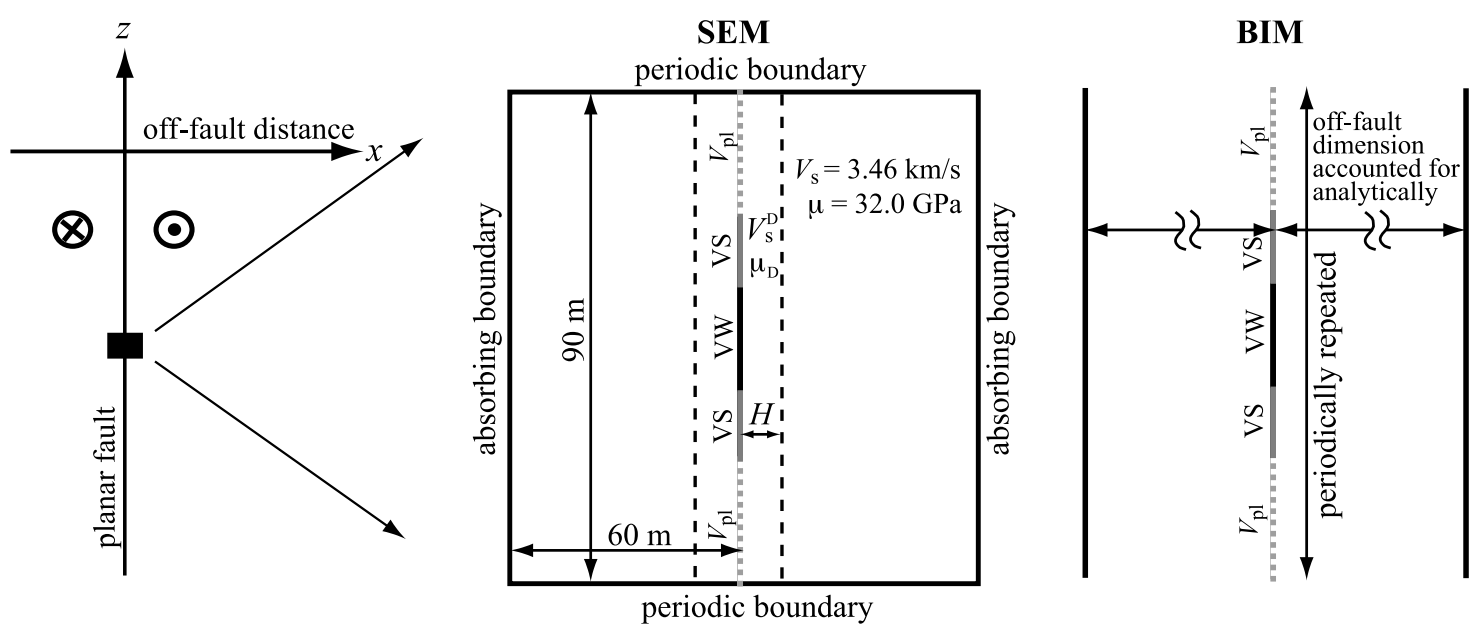

Figure 2. 2-D models of a vertical strike-slip fault. Small repeating earthquakes at seismogenic depths in a region indicated by a black rectangle are simulated using these models. The fault is $90-\mathrm{m}$ long. Slip evolution is computed, based on the assumed friction law, on the $45-\mathrm{m}$ long central portion, and the prescribed slip rate $V_{\mathrm{pl}}=2 \mathrm{~mm} / \mathrm{yr}$ is imposed on the two $22.5-\mathrm{m}$ long outer portions. The fault is divided into three segments: a 19-m long central velocity-weakening (VW) patch surrounded by two 13-m long velocity-strengthening (VS) regions. By symmetry consideration, the medium across the fault boundary has equal and opposite motion. Unless otherwise noted, the medium is assumed to be homogeneous. In section 4 , we investigate the effect of a fault-parallel low-rigidity layer of a width $H$ and rigidity $\mu_{\mathrm{D}}$ on seismic and aseismic slip.

involves another conjugate gradient solution, with $\mathbf{u}_{n+1}^{* \mathrm{~m}}$ as an initial guess.

[25] 8. Make the corresponding prediction $\tau_{n+1}^{\mathrm{tot} * *}$ and $\theta_{n+1}^{* *}$ by repeating steps 3 and 4 and by replacing $\dot{\delta}_{n}$ in equation (20) or (21) with $\left(\dot{\delta}_{n}+\dot{\delta}_{n+1}^{*}\right) / 2$.

[26] 9. Find the final prediction $\dot{\delta}_{n+1}^{* *}$ and the components of $\dot{\delta}_{n+1}^{* *}$ by repeating step 5 with $\tau_{n+1}^{\text {tot*** }}$ and $\theta_{n+1}^{* *}$ instead of $\tau_{n+1}^{\text {tot } *}$ and $\theta_{n+1}^{*}$.

[27] 10. Declare the values of $\dot{\delta}_{n+1}, \theta_{n+1}$, and $\tau_{n+1}^{\text {tot }}$ on the fault, and the values of displacement of the entire medium $\mathbf{u}_{n+1}$, to be equal to the predictions with the superscript double asterisks.

[28] In steps 2 and 7, we terminate the conjugate gradient iteration when $\|$ LHS - RHS $\|/\| \mathrm{RHS} \|<\varepsilon_{\mathrm{CG}}=10^{-5}$, where LHS and RHS are the left-hand side and right-hand side of equation (18), respectively. The convergence rate in the conjugate gradient iteration greatly depends on the type of preconditioner. Currently, we use the Jacobi preconditioner, one of the simplest forms of preconditioning, for the conjugate gradient method. For SEMs, a special preconditioner called 'the Schwarz method' has been shown to significantly increase the convergence rate for elastic problems [Lottes and Fischer, 2004; Zampieri and Pavarino, 2006]. Implementing the Schwarz preconditioner remains a subject of future work.

\section{Implementation Example}

\subsection{Formulation of a 2-D Model}

[29] The response of faults to tectonic loading is characterized by long periods of quasi-static deformation combined with short periods of fast dynamic slip. To simulate such response, we adopt the variable time stepping procedure of Lapusta et al. [2000], in which the time step is set to be inversely proportional to slip velocity on the fault as described in Appendix C. As a result, relatively large time steps, a fraction of a year, are used in the interseismic periods, while small time steps, a fraction of a second or smaller, are used to simulate fast seismic slip. Note that the efficiency of the time stepping procedure (Appendix C) depends on the degree of the positive direct effect in the rate and state formulation [Lapusta et al., 2000], a feature that has ample laboratory confirmation.

[30] The updating scheme introduced in the previous section can be merged with the explicit time stepping scheme of the fully dynamic SEM problem (Appendix B). The main challenge is to find proper criteria for switching from the quasi-static implicit scheme to the dynamic explicit scheme and vice versa. At the onset of earthquakes (or at the end of nucleation processes), slip velocities abruptly increase from values much smaller than typical plate loading rates $\left(\sim 10^{-10}-10^{-9} \mathrm{~m} / \mathrm{s}\right)$ to coseismic values $(\sim 1-10 \mathrm{~m} / \mathrm{s})$, and the time step progressively becomes smaller. Hence we switch from one scheme to the other based on the values of the maximum slip velocity. For the problems discussed below, we switch from the quasi-static to dynamic scheme at $\dot{\delta}_{\max }^{\mathrm{QD}}=0.5 \mathrm{~mm} / \mathrm{s}$ and from the dynamic to quasi-static scheme at $\dot{\delta}_{\max }^{\mathrm{DQ}}=0.2 \mathrm{~mm} / \mathrm{s}$. Ideally, one could formulate a switching criterion based on the relative importance of the inertial term in the governing equation (2). However, we find that such quantity is numerically more unstable than the criteria based on the values of the maximum slip velocity (Appendix D). We confirm that the criteria we adopt here ensures that, at the times of the switch, the inertial term in the governing equation is much smaller $\left(\sim 10^{-6}\right)$ relative to the other terms (Appendix D) and that the results compare well with BIM methods as discussed below.

[31] To demonstrate how the ideas outlined so far are combined to produce long-term deformation histories, let us consider the response of a 2-D model of a vertical strike-slip 
(a)

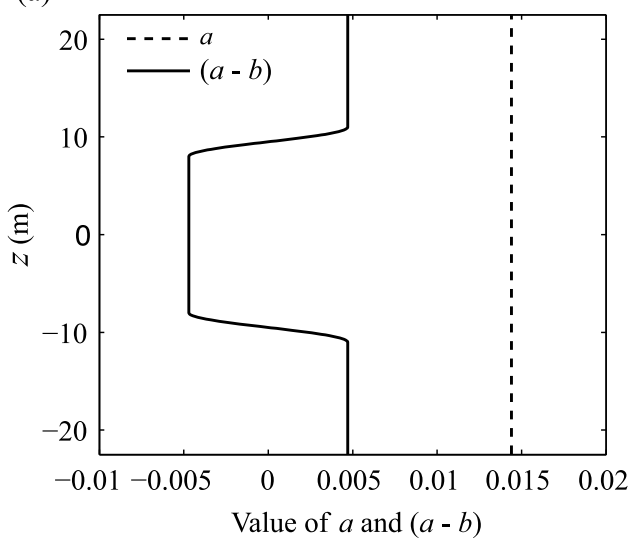

(b)

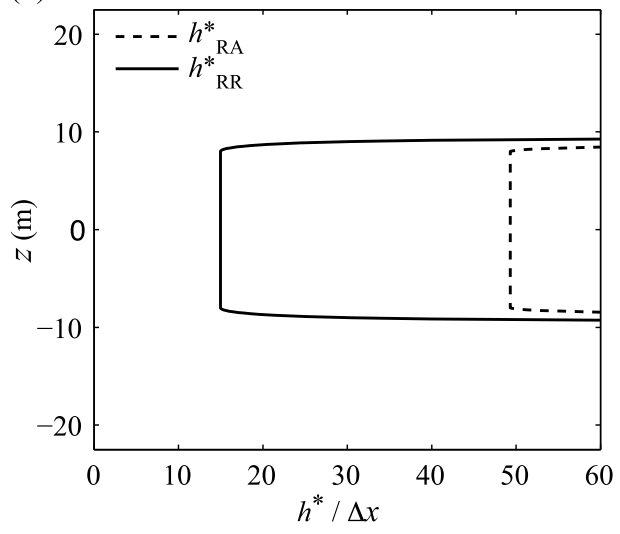

Figure 3. (a) Depth-variable distribution of friction parameters $a$ and $(a-b)$. (b) Distribution of the ratio $h^{*} / \Delta x$. Two theoretical estimates $h^{*}$ of the nucleation size by Rubin-Ampuero (RA) and Rice-Ruina (RR) are shown.

fault embedded in an elastic medium (Figure 2). On the fault, a potentially seismogenic patch borders regions steadily moving with the prescribed slip rate $V_{\mathrm{pl}}=2 \mathrm{~mm} / \mathrm{yr}$, as illustrated in Figure 2. That steady motion provides loading. The fault motion is in the along-strike direction $y$, but only variations with depth $z$ are considered, so that the fault behavior is described by strike-parallel slip $\delta(z, t)$, slip velocity (or slip rate) $\dot{\delta}(z, t)=\partial \delta(z, t) / \partial t$, and the relevant component of shear stress $\tau^{\text {tot }}(z, t)$. The symmetries of the problem allow us to restrict the computational domain to the medium on one side of the fault $(x \geq 0)$.

[32] It is convenient to express the formulae in terms of variables $\left(u(x, z, t)-V_{\mathrm{pl}} t / 2\right)$ and $\left(\dot{u}(x, z, t)-V_{\mathrm{pl}} / 2\right)$, in which case $\tau_{\mathrm{o}}(x, z, t)$ becomes independent of time and equal to the initial stress $\tau_{\mathrm{o}}(x, z)$. This approach was used for the BIM model of Lapusta et al. [2000]. For the 2-D problems we consider here, the medium across the fault boundary has equal and opposite motion by symmetry consideration. Then the relation (9) on the fault becomes

$$
\begin{aligned}
\tau^{\mathrm{tot}}(z, t)= & \tau_{\mathrm{o}}(z)-\frac{1}{2 B(z)}\left(K_{11}(y, z, t)\left[u^{\mathrm{f}}(z, t)-\frac{V_{\mathrm{pl}} t}{2}\right]\right. \\
& \left.+K_{12}(y, z, t)\left[u^{\mathrm{m}}(z, t)-\frac{V_{\mathrm{pl}} t}{2}\right]\right) .
\end{aligned}
$$

Note that our mesh is conformal and hence $B=B_{+}=B_{-}$.

[33] The SEM model consists of a $90 \mathrm{~m}$ by $60 \mathrm{~m}$ rectangular domain (Figure 2). To allow comparison to the BIM, the domain is replicated using periodic boundary conditions on both sides of the domain (Figure 2). The fault boundary obeys rate and state friction with the aging law (11). The model contains variations in steady state friction properties that create rheological transitions (Figure 3). The parameters used in the simulations are listed in Table 1 . The effective normal stress $\bar{\sigma}$ and characteristic slip $L$ are uniform along the fault.

[34] We use the criteria for spatial discretizations developed in the work by Perfettini and Ampuero [2008] and Lapusta and Liu [2009], which showed that resolving a cohesive zone size is a more stringent requirement than resolving the nucleation size, for the aging formulation of rate and state friction and typical rate and state parameters. The spatial discretization required to resolve dynamic rup- ture is largely sufficient for properly resolving the interseismic deformation. An optimal mesh for the quasi-static problem would involve mesh coarsening as a function of distance from the fault. Here we do not consider such optimization and, to avoid mapping between different meshes, we adopt the same mesh for both the dynamic and quasi-static problems. We discretize the domain into quadrilateral spectral elements with an average node spacing $\Delta x=0.25 \mathrm{~m}$ in each element. Note that for SEMs, the nodes are generally nonuniformly distributed, and hence we report the node spacing in terms of their average value. The average node spacing $\Delta x=0.25 \mathrm{~m}$ results in $\Lambda_{\mathrm{o}} / \Delta x \approx$ $\mu L /(b \bar{\sigma} \Delta x) \approx 5$ where $\Lambda_{\mathrm{o}}$ is the cohesive zone size at the rupture speed $V_{\mathrm{r}} \rightarrow 0^{+}$. Such resolution has shown to be adequate in the work of Day et al. [2005] and Lapusta and Liu [2009], and it leads to stable results in our simulations that do not change due to finer discretizations. The selected spatial discretization corresponds to $h_{R A}^{*} / \Delta x \approx 50$ (Figure 3b), where $h_{R A}^{*}$ is the estimate of the nucleation size obtained by Rubin and Ampuero [2005] for $a / b \gtrsim 0.5$, see equation (16).

\subsection{Comparison of Simulation Results Obtained With 2-D SEM and 2-D BIM}

[35] To assess the accuracy of numerical results, we conduct comparison of simulation results obtained using the developed SEM model with those of the BIM spectral formulation of Lapusta et al. [2000], which resolves all stages of each earthquake episode under a single computational scheme. Figure 2 illustrates the geometry of the antiplane

Table 1. Parameters Used in the 2-D SEM and 2-D BIM Models of Small Repeating Earthquakes

\begin{tabular}{lcc}
\hline \multicolumn{1}{c}{ Parameter } & Symbol & Value \\
\hline Shear modulus & $\mu$ & $32.0 \mathrm{GPa}$ \\
Shear wave speed & $V_{\mathrm{s}}$ & $3.464 \mathrm{~km} / \mathrm{s}$ \\
Reference slip rate & $\dot{\delta}_{0}$ & $10^{-6} \mathrm{~m} / \mathrm{s}$ \\
Reference friction coefficient & $f_{0}$ & 0.6 \\
Characteristic slip distance & $L$ & $84.0 \mathrm{micron}$ \\
Effective normal stress & $\bar{\sigma}$ & $120 \mathrm{MPa}$ \\
Rate and state parameter $a$ & $a$ & 0.0144 \\
Rate and state parameter $b$ & $b$ & $0.0191^{\mathrm{a}}$ \\
\hline
\end{tabular}

${ }^{\text {a }}$ The indicated value of $b$ is for the velocity-weakening region in Figure 2. 
(a)

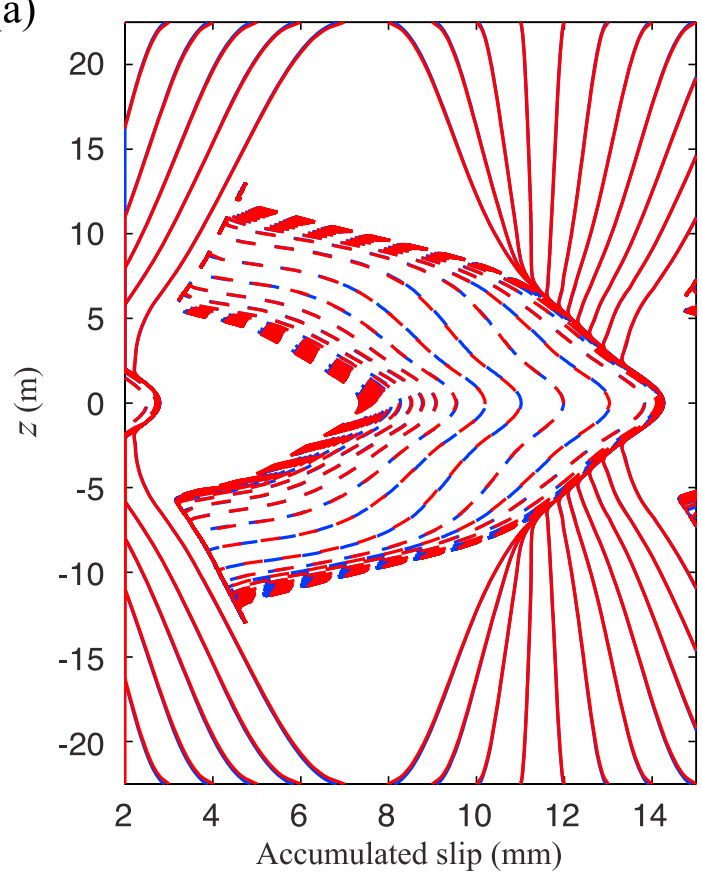

(b)

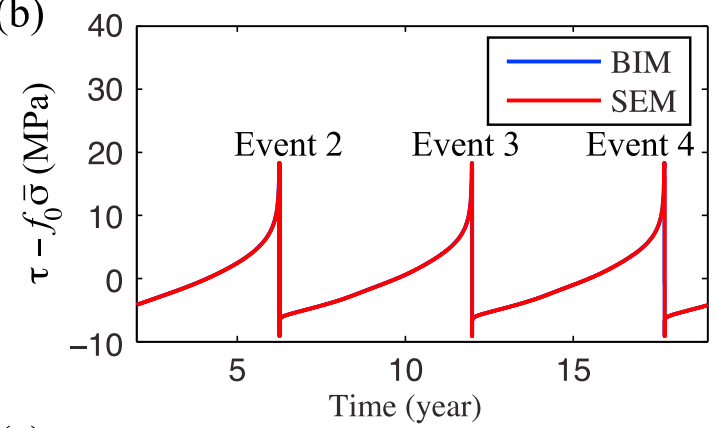

(c)

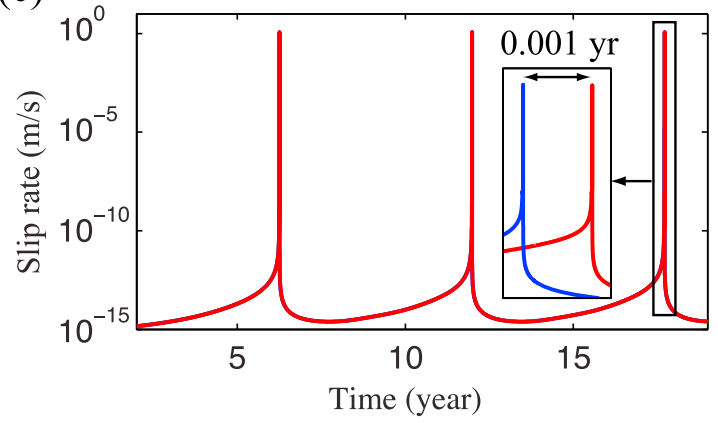

Figure 4. Comparison of earthquake sequences simulated in BIM and the developed SEM. (a) Solid lines show slip accumulation every 0.5 years for BIM (blue) and SEM (red). Dashed lines are intended to capture dynamic events and are plotted every 1 millisecond during the simulated earthquakes (with $\dot{\delta}_{\max }>1 \mathrm{~cm} / \mathrm{s}$ ). Evolution of aseismic slip and seismic slip of the 2 nd event are shown. Spatial distributions of slip contours in these models agree very well. (b) Shear-stress and (c) slip-velocity histories at the center of the fault. The $2 \mathrm{nd}$, 3rd, and 4th earthquake events are shown. The timings of earthquake events in these models are nearly identical as quantified in the text, verifying our SEM implementation.

SEM and BIM models. In BIM, wave propagation is analytically accounted for by boundary integral expressions. The method assumes the fault is repeated periodically, which we also enforce in the SEM model.

[36] Earthquake sequences simulated in SEM and BIM models are shown and compared in Figure 4. There are four seismic events in the sequence. Assuming that the source dimension is the same for the along-strike and along-dip directions, the moment magnitude of each seismic event is $\mathrm{M}_{\mathrm{w}}$ 1.3. The solid lines are plotted every 0.5 years and show the continuous slow sliding (creep) of the velocitystrengthening regions. That slow slip creates stress concentration at its tip and penetrates into the velocity-weakening region. In due time, an earthquake rupture nucleates and propagates bilaterally; its progression is shown by dashed lines. After an earthquake arrests, the velocity-strengthening region experiences accelerated sliding, or afterslip, due to the transferred stress. The interseismic period between two successive events is about 6 years.

[37] The overall agreement of spatial slip distributions between two models during coseismic as well as interseismic periods verifies our developed SEM approach (Figure 4a). The histories of shear stress and slip velocity at the center of the fault in these models are virtually identical, and the timing of the onset $\left(\dot{\delta}_{\max }>1 \mathrm{~cm} / \mathrm{s}\right)$ of the 4 th seismic event in these models differs by $0.005 \%$ (Figures $4 \mathrm{~b}$ and $4 \mathrm{c})$. The agreement is very good given that, in the SEM simulation, there are a total of $\sim 40,000$ adaptive time steps, each of which includes 1 to 500 conjugate gradient itera- tions. To make sure that the solution is accurate, we have checked that the result of a BIM simulation with the twice higher resolution shows identical slip patterns and timings of seismic events, confirming that our results have converged to reasonable accuracy.

[38] Figure 5 shows the evolution of the velocity fields during the interseismic and coseismic periods in the SEM model. About one year before the seismic event, the velocity-weakening segment is only partly locked. About one day before, preseismic deformation due to the gradually accelerating nucleation process starts to show up near the fault. About one minute before, the actively slipping region on the fault is comparable to the eventual nucleation size and the corresponding near-fault deformation becomes greater. The change in strain field associated with this deformation can be detected if a strainmeter were placed at an off-fault distance comparable to the size of the nucleation region. However, borehole strainmeters are generally located near the Earth surface, and hence observations of premonitory slip prior to the eventual main shock are difficult [Tullis, 1996]. The edges of the nucleation zone dynamically accelerate in the opposite direction, leading to bilateral rupture during the seismic event (Figure 5b). After the seismic event that occurred between the 4th and 5th panels in Figure 5a, the white and yellow region gradually expands due to afterslip on the velocity-strengthening segments of the fault. The afterslip terminates about one year after the seismic event and the velocity-weakening fault segment is locked again. 

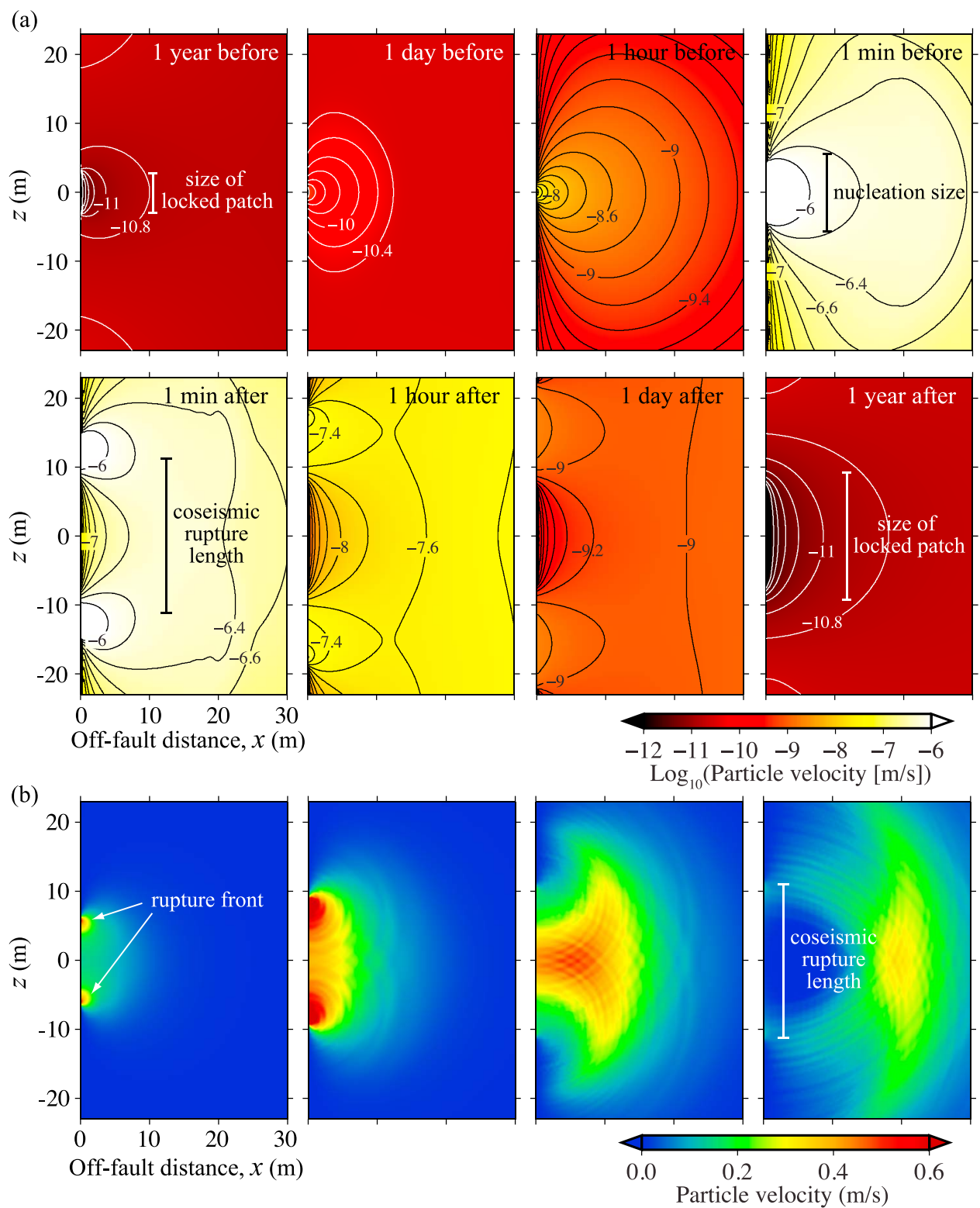

Figure 5. (a) Snapshots of the SH particle velocity field during the interseismic periods. Colors represent particle velocity, with preseismic (i.e., nucleation) and postseismic deformation in white and yellow, the loading rate $0.5 V_{\mathrm{pl}}$ in dark red, and deformation associated with fault locking shown in black. Contours correspond to the values of $\log _{10}$ (velocity $[\mathrm{m} / \mathrm{s}]$ ). The line $x=0$ corresponds to the fault. (b) Snapshots of the velocity field every 3 milliseconds during the seismic event.

[39] Since the quasi-static scheme involves solving a large linear system (6), interseismic deformation associated with nucleation, afterslip, and fault creep requires much more computational time than dynamic-rupture and wave propagations do. For the problems we consider, more than $95 \%$ of the CPU time is spent on computing the interseismic deformation. Hence future work is directed toward optimizing the quasi-static solution scheme to improve its performance.

\section{Effects of a Fault-Parallel Low-Rigidity Layer on Seismic and Aseismic Slip}

[40] We use the SEM model developed in section 2 and verified in section 3 to investigate the effects of variable bulk properties on repeating earthquakes. The model set up is similar to the one shown in Figure 2 except that a fault parallel low-rigidity layer of width $H$ and rigidity $\mu_{\mathrm{D}}$ is added in the vicinity of the fault, to mimic a localized damaged fault zone. The friction-related parameters and the distribution of effective normal stress are the same as in section 3. We examine how earthquake source properties, such as stress drop, recurrence intervals, and nucleation sizes, depend on the width of the low-rigidity layer.

[41] Figure 6 shows simulated earthquake sequences for two scenarios: a case with a low-rigidity layer $\left(\mu_{\mathrm{D}}=20.5 \mathrm{GPa}\right)$ of width $H=1.5 \mathrm{~m}$ and the other with a homogeneous bulk with rigidity $\mu_{\mathrm{D}}=20.5 \mathrm{GPa}(H=$ infinity $)$. Note that the rigidity ratio is $\mu_{\mathrm{D}} / \mu=0.64$ and the corresponding ratio of the $S$-wave 

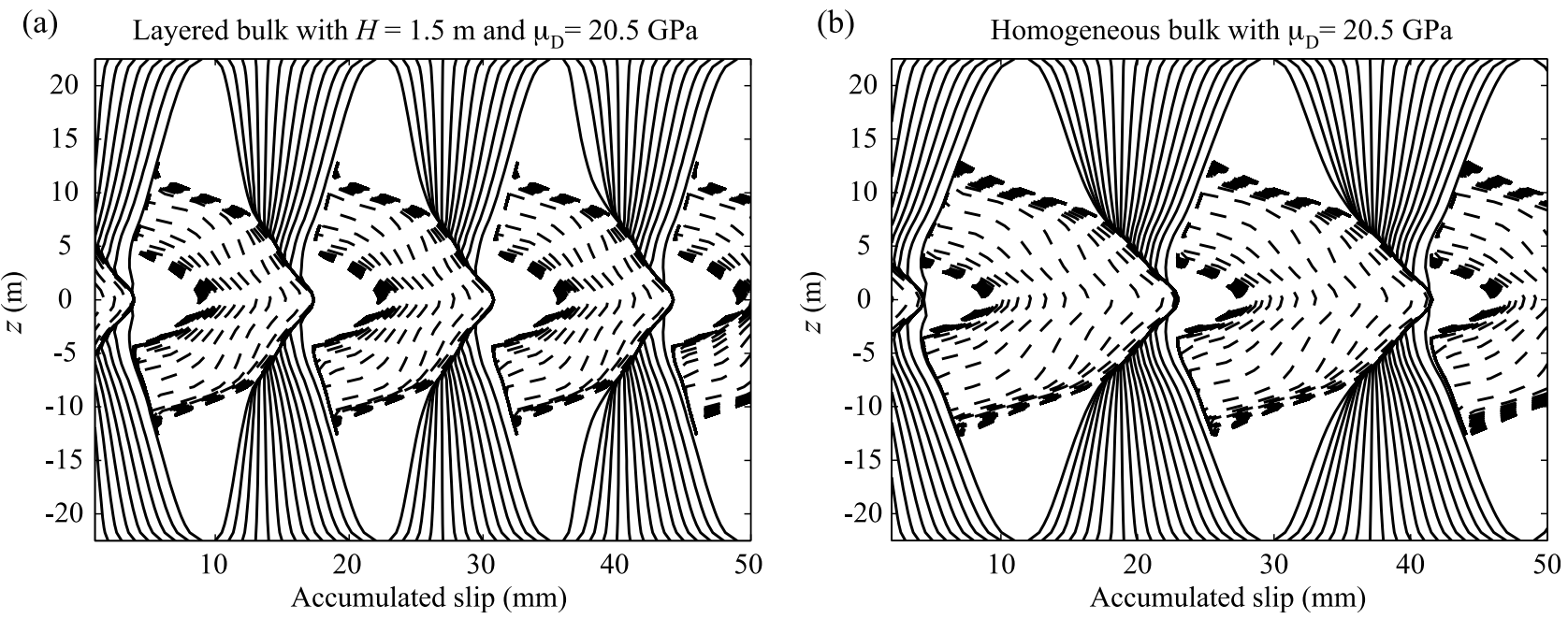

Figure 6. Simulated earthquake sequences with (a) layered bulk structure with the width of a low-rigidity layer $H=1.5 \mathrm{~m}$ and (b) homogeneous bulk structure with $\mu_{\mathrm{D}}=20.5 \mathrm{GPa}$ corresponding to $H=\infty$. Solid and dashed lines have the same meaning as in Figure 4a. The model geometry is shown in Figure 2.

speeds is $V_{\mathrm{s}}^{\mathrm{D}} / V_{\mathrm{s}}=0.8$. As Figure 4a and Figure 6 show, the earthquake source properties are affected by the width of the low-rigidity layer. We further perform several simulations with different values of $H$ and quantify the dependence of earthquake source properties on $H$ (Figure 7). In the following, we summarize several key findings.

\subsection{Reduction of Nucleation Sizes in Low-Rigidity Fault Zones}

[42] The theoretical estimates of a nucleation size given in equations (15) and (16) predict that the nucleation size on a planar fault embedded in a homogeneous medium is linearly proportional to rigidity $\mu$ of the medium. This is consistent with our simulation results for the scenarios with homogeneous bulk (Figure 7a). To compute nucleation sizes in our simulations we use a criterion based on rupture speed: we define the onset of instability as the time when a tip of the actively slipping zone moves with the speed that exceeds a fraction $(10 \%)$ of the shear wave speed of the surrounding elastic medium [Kaneko and Lapusta, 2008]. The tips of the actively slipping zone are found as the locations of peak shear stress.

[43] The dependency of nucleation size on fault zone width $H$ has two extreme regimes. The nucleation size approaches the length $h^{*}$ estimated with the rigidity $\mu$ of the undamaged host rock when $H$ is small compared to that length. As $H$ increases, the influence of the low-rigidity layer on the nucleation size becomes greater. The nucleation size approaches the length $h^{*}$ estimated with the rigidity $\mu_{\mathrm{D}}$ of the fault zone damaged rock when $H$ is a large fraction of that length.

[44] In the transition between these two extreme regimes, linear stability analysis (Appendix E) provides a theoretical estimate of the nucleation size $h_{\text {lay }}^{*}$ on rate and state faults embedded in a simple layered medium, given as the solution of the following equation (E3):

$$
h_{\text {lay }}^{*} \tanh \left[H \frac{\pi}{2 h_{\text {lay }}^{*}}+\operatorname{arctanh}\left(\frac{\mu_{\mathrm{D}}}{\mu}\right)\right]=h_{\text {hom }}^{* \mu_{\mathrm{D}}},
$$

where $h_{\mathrm{hom}}^{* \mu_{\mathrm{D}}}$ is the estimate of a nucleation size in a homogeneous medium with rigidity $\mu_{\mathrm{D}}$. The theoretical prediction of $h_{\text {lay }}^{*}$ in equation (25) obtained by setting $h_{\text {hom }}^{* \mu_{\mathrm{D}}}=h_{\mathrm{RA}}^{* \mu_{\mathrm{D}}}$, where $h_{\mathrm{RA}}^{*}$ is given by equation (16), agrees fairly well with the simulated nucleation sizes for a range of $H$ (Figure 7a).

[45] Using friction parameters found in laboratory experiments of Blanpied et al. [1995], $b-a=0.004, a=0.01$ and $L=1-10$ microns, and assuming that $\bar{\sigma}=100 \mathrm{MPa}$ at seismogenic depth, the nucleation size is of the order of $0.1-1 \mathrm{~m}$ based on equations (15) and (16). This suggests that a nucleation size for natural earthquakes that occur on faults embedded in meter-scale damaged but still cohesive rock are controlled by the properties of the damaged rock and can be smaller than the value estimated using the rigidity of undamaged rocks.

\subsection{Dynamic Amplification of Slip Rates and Slip} in Low-Rigidity Fault Zones

[46] As the width of a low-rigidity layer $H$ becomes larger, the peak slip rate of the propagating dynamic rupture amplifies (Figure 7b). As a result, both the average and peak coseismic slip also increases with $H$ (Figure 7c). Between the end-member cases with homogeneous bulk $\mu$ and $\mu_{\mathrm{D}}$, the amplification of the peak slip rate and peak slip in Figures $7 \mathrm{~b}$ and $7 \mathrm{c}$ is about 3.0 and 2.0 , respectively, larger than the ratio of the rigidity contrast $\mu / \mu_{\mathrm{D}}=1.56$. To understand this behavior, we consider an analytical solution for the maximum slip rate due to a propagating shear crack at a constant speed in a homogeneous medium. In this case, the peak slip rate $\dot{\delta}_{\text {max }}$ and the rupture speed $V_{\mathrm{r}}$ are related by $[$ Ida, 1973]:

$$
\dot{\delta}_{\max } \propto \frac{V_{\mathrm{r}} \Delta \tau^{\mathrm{p}-\mathrm{s}}}{\mu \Lambda_{\mathrm{III}}},
$$

where $V_{\mathrm{r}}$ is a rupture speed of the propagating shear crack, $\Delta \tau^{\mathrm{p}-\mathrm{s}}$ is the change from the peak stress to the dynamic sliding stress (i.e., strength drop), and $\Lambda_{\mathrm{III}}\left[V_{\mathrm{r}} / V_{\mathrm{s}}\right]=\left(1-V_{\mathrm{r}}^{2} /\right.$ $\left.V_{\mathrm{s}}^{2}\right)^{1 / 2}$ is a monotonically decreasing universal function of $V_{\mathrm{r}}$. 
(a)

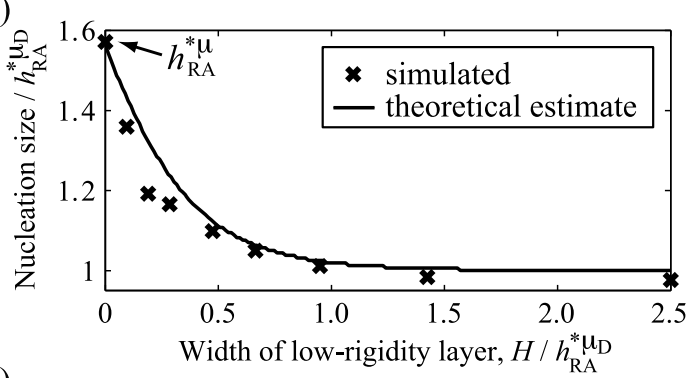

(c)

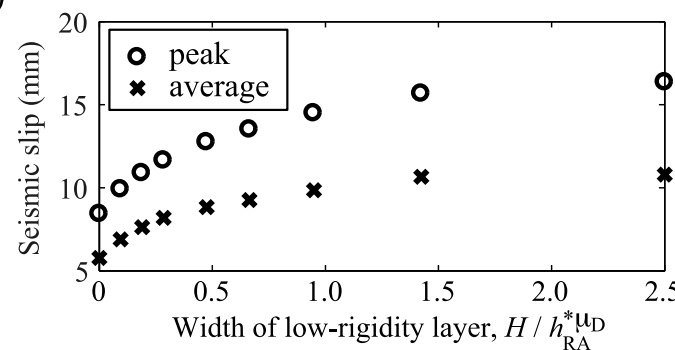

(e)

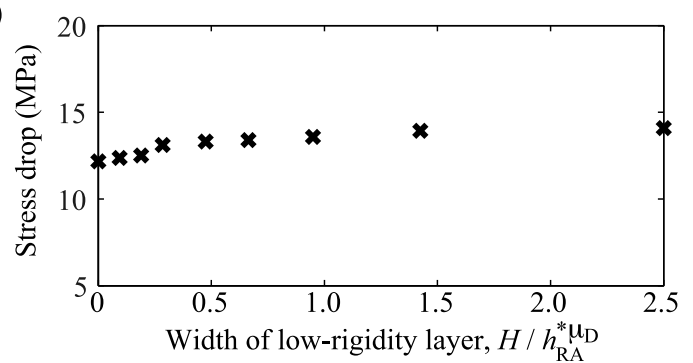

(g)

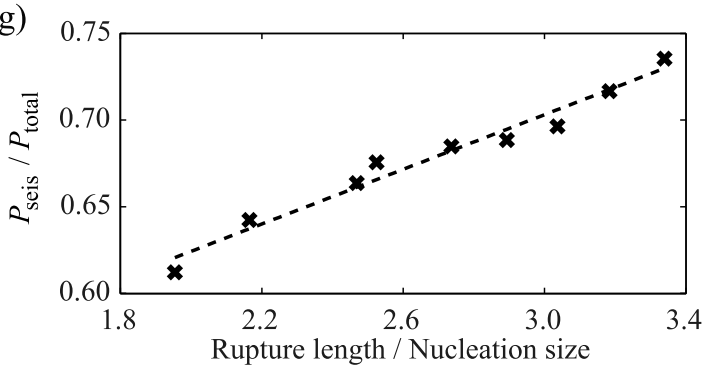

(b)

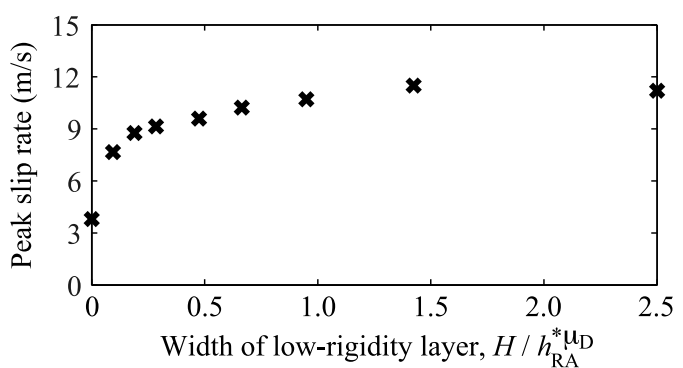

(d)
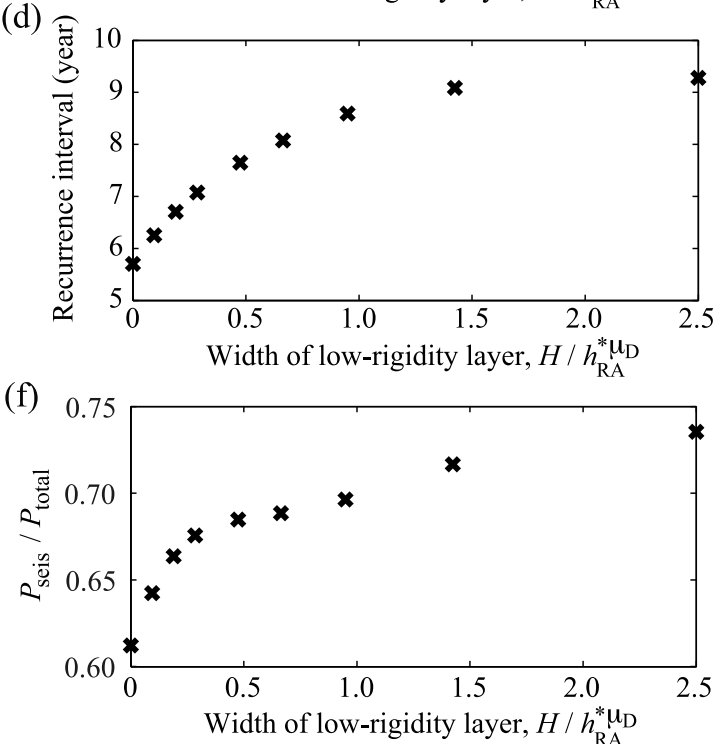

(h)

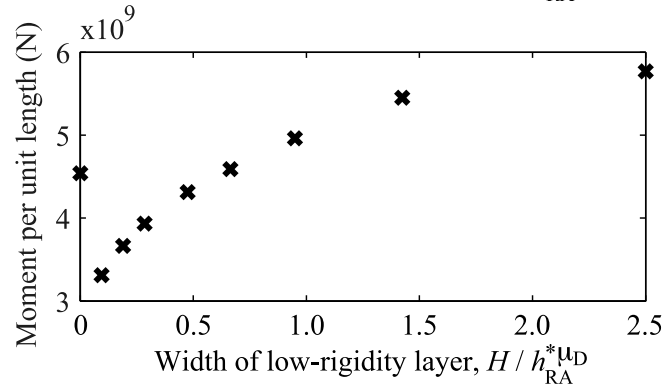

Figure 7. Effects of a fault parallel low-rigidity layer of width $H$ on small repeating earthquakes. (a) The relation between simulated nucleation sizes and $H$. The nucleation sizes and the widths are nondimensionalized by the theoretical estimate $h_{R A}^{* \mu_{\mathrm{D}}}$, with $h_{R A}^{* \mu_{\mathrm{D}}}=7.9 \mathrm{~m}$ for the parameters used. The solid curve is the theoretical prediction (25), which fits the simulated nucleation sizes fairly well. (b) The peak slip rate during a typical earthquake as a function of $H$. (c) The peak coseismic slip and the averaged slip over the source as a function of $H$. (d) Recurrence interval between successive earthquakes as a function of $H$. (e) Stress drop averaged over the region of positive stress drop as a function of $H$. (f) Ratio of seismic potency $P_{\text {seis }}$ to total potency $P_{\text {total }}$ released on the velocity-weakening patch over one earthquake cycle. (g) $P_{\text {ctseis }} / P_{\text {total }}$ as a function of the effective rupture dimension (i.e., the ratio of the rupture length to the simulated nucleation size). The dashed line is the least squares fit to the data points. The increase of the effective rupture dimension due to increasing $H$ leads to the increase of $P_{\text {seis }} / P_{\text {total }}$ and hence more seismic slip. (h) The seismic moment per unit length as a function of $H$. The case with $H / h_{R A}^{*} \mu_{\mathrm{D}}=2.5$ in Figures $7 \mathrm{a}-7 \mathrm{f}$ and $7 \mathrm{~h}$ corresponds to the case for a homogeneous bulk structure with $\mu_{\mathrm{D}}=20.5 \mathrm{GPa}$ shown in Figure $6 \mathrm{~b}$.

We find that, in our simulations, the strength drop $\Delta \tau^{\mathrm{p}-\mathrm{s}}$ does not depend much on the rigidity $\mu$ of the medium. The ratio of the peak slip rate between damaged and undamaged fault zone scenarios then becomes

$$
\frac{\dot{\delta}_{\max }^{\mathrm{D}}}{\dot{\delta}_{\max }^{\text {hom }}}=\frac{V_{\mathrm{r}}^{\mathrm{D}}}{V_{\mathrm{r}}^{\text {hom }}} \frac{\Lambda_{\mathrm{III}}\left[V_{\mathrm{r}}^{\text {hom }} / V_{\mathrm{s}}\right]}{\Lambda_{\mathrm{III}}\left[V_{\mathrm{r}}^{\mathrm{D}} / V_{\mathrm{s}}^{\mathrm{D}}\right]} \frac{\mu}{\mu_{\mathrm{D}}},
$$

where the quantities with the superscript 'hom' refer to the case for the undamaged fault zone (a homogeneous bulk).

[47] Figure $8 \mathrm{~b}$ shows a comparison between the theoretical prediction (27) and the ratio of the simulated peak slip rate in the damaged fault zone cases to that in the homogeneous case with $\mu=32 \mathrm{GPa}$ (Figure $7 \mathrm{~b}$ ). We set rupture speed $V_{\mathrm{r}}^{\mathrm{D}}$ in expression (27) to be the maximum value of $V_{\mathrm{r}}^{\mathrm{D}}$ 
(a)

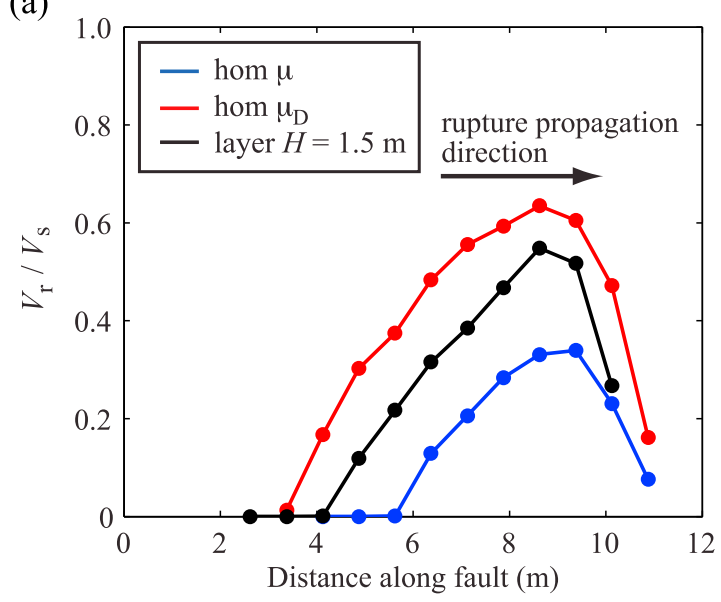

(b)

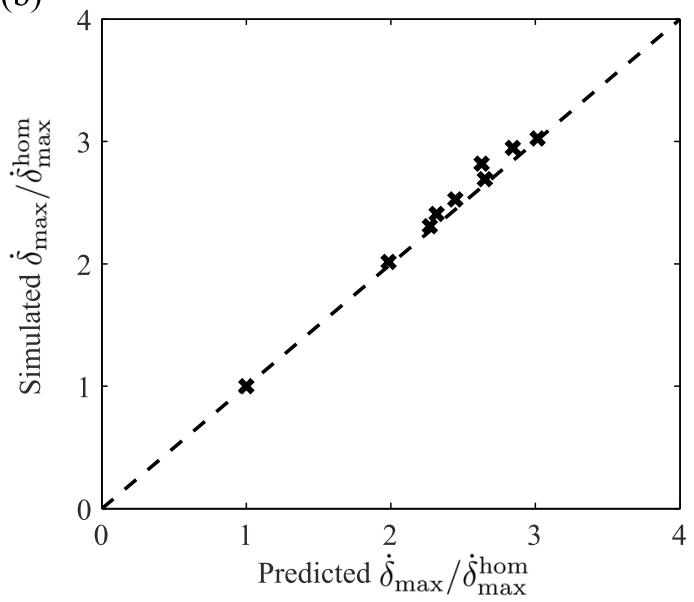

Figure 8. (a) Rupture speeds $V_{\mathrm{r}}$ normalized by the $S$-wave speed $V_{\mathrm{s}}$ of the medium adjacent to the fault during a seismic event for the cases with a homogeneous bulk $\mu$ and $\mu_{\mathrm{D}}$ and for a layered case with $H=$ $1.5 \mathrm{~m}$. Rupture speed is determined by computing average rupture speed over each spectral element (or 5 computational nodes) and plotting the obtained value with respect to the center of the element. The rupture speeds become larger for a larger width $H$ of a low-rigidity layer due to the increase in the effective rupture dimension. (b) The peak slip rate $\dot{\delta}_{\max }$ shown in Figure $7 \mathrm{~b}$ divided by that in the case for a homogeneous bulk with $\mu=32 \mathrm{GPa}\left(\dot{\delta}_{\max }^{\text {hom }}\right)$. The ratio of the peak slip rates $\dot{\delta}_{\max } / \dot{\delta}_{\max }^{\text {hom }}$ predicted by equation (27) is in good agreement with the ratio of the simulated peak slip rates.

during a seismic event in each case (Figure 8a) because the slip rate and rupture speed are generally correlated. The good agreement between the simulations and the theoretical prediction in Figure $8 \mathrm{~b}$ suggests that the higher peak slip rates are caused by a combination of the rigidity contrast and the difference in effective rupture dimension, i.e., the ratio of the rupture length to the nucleation size. In our models, both the peak slip rate and rupture speed increase as the rupture propagates a longer distance (Figure 8a). Since the effective rupture dimension is larger in the damaged fault zone due to the reduction of the nucleation size (Figure 7a), the resulting rupture speeds are higher. This effect further amplifies the peak slip rate and slip in the damaged fault zone cases in addition to the rigidity contrast. This result suggests that the peak slip rates of small repeating earthquakes in severely damaged and undamaged rocks may be different by an order of magnitude and the corresponding seismic slip would become significantly larger.

\subsection{Larger Recurrence Interval in Low-Rigidity Fault Zones}

[48] Interestingly, the recurrence interval of the simulated earthquakes increases as the width of a lower-rigidity layer increases (Figure 7d). One may think that a smaller nucleation size in the case with the low-rigidity bulk would reduce the recurrence interval, but the opposite happens. To understand this, we consider a simpler model of an earthquake sequence with constant stress drop $\Delta \tau$ and constant stressing rate $\dot{\tau}$. The recurrence interval $T_{\mathrm{r}}$ in this scenario is given by $T_{\mathrm{r}}=\Delta \tau / \dot{\tau}$. Figure $7 \mathrm{e}$ shows that the stress drop slightly increases with increasing width of a low-rigidity layer due to the dynamic amplification of slip rates. However, this effect alone cannot fully explain the greater increase of the recurrence interval. We find that the difference in interseismic stressing rates caused by different rigidity of the adjacent rock contributes to the increase of the recurrence interval. Since our model is loaded by the back-slip motion $V_{\mathrm{pl}}$ outside of the velocity-strengthening fault segments and the corresponding stressing rate on the fault strongly depends on the rigidity of the medium adjacent to the fault plane, $\dot{\tau}$ is smaller for the lower-rigidity bulk medium. This is why the recurrence interval is larger for the cases with the lower-rigidity medium. Since tectonic loading is applied quasi-statically, the dependence of the recurrence interval on the width of a low-rigidity layer is similar to that of the nucleation size.

\subsection{Smaller Amount of Aseismic Slip in Low-Rigidity Fault Zone}

[49] To quantify the amount of the aseismic slip compared to the seismic slip during one earthquake cycle, we compute seismic and total potency in the 2-D model. Potency can not be usefully discussed in the 2-D model where slip extends infinitely, and simultaneously, along strike at any given depth $z$. We therefore express potency in terms of potency per unit length and compute it as follows:

$$
P=\int_{\mathrm{VW} \text { patch }} \delta(z) d z,
$$

where $P$ is computed over the velocity-weakening (VW) patch.

[50] The ratio of the seismic potency to the total potency $P_{\text {seis }} / P_{\text {total }}$ increases as the width of a low-rigidity layer $H$ increases (Figure 7f). This means that more seismic slip is promoted in the bulk with the lower-rigidity layer. When $H$ is comparable to the source dimension (i.e., the ruptured length), the ratio $P_{\text {seis }} / P_{\text {total }}$ approaches the value for the case with a homogeneous bulk with $\mu_{\mathrm{D}}$. We find that the increase of $P_{\text {seis }} / P_{\text {total }}$ for larger $H$ can be explained by the increase in the effective rupture dimension, i.e., the ratio of the rupture length to the nucleation size (Figure 7g). A smaller nucleation size and the resulting larger rupture dimension for 
(a)

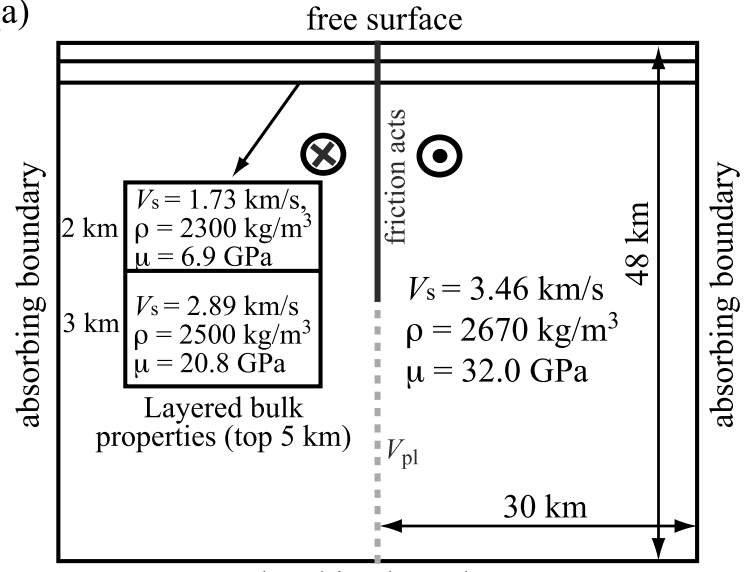

(b)

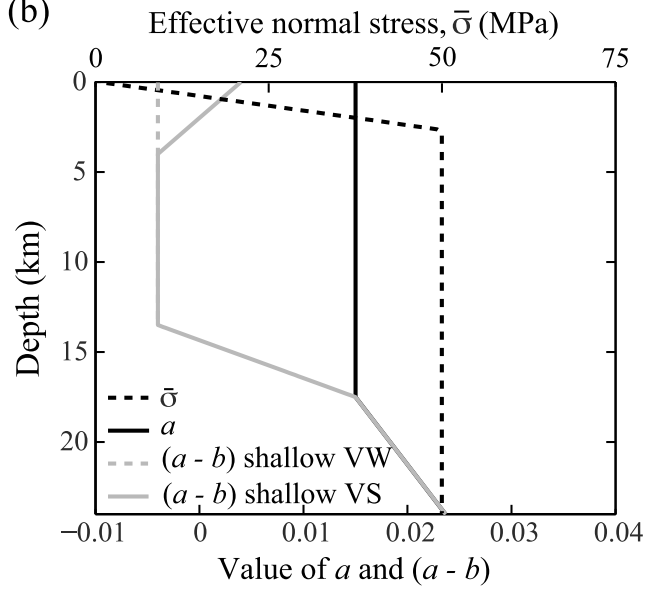

Figure 9. (a) 2-D SEM model of a vertical strike-slip fault. Layered bulk properties used in some scenarios we consider are indicated. (b) Depth-variable distribution of effective normal stress, the rate-andstate constitutive parameters $a$ and $(a-b)$ over the fault segment where friction acts.

larger $H$ leads to a smaller amount of aseismic slip over the earthquake cycle.

[51] We note that the presence of heterogeneity in bulk properties leads to an ambiguity in the notion of the seismic moment. If the local, near-fault value of rigidity is used for computing the seismic moment, then there is a sudden change between $H=0$ and a small non-zero $H$ (Figure 7h), due to the change in the rigidity value used. This is problematic since there is no discontinuity in a physical behavior as $H$ approaches 0 . At the same time, the seismic moment inferred from far-field observations of long-period waves would reflect the value of rigidity for the large-scale, undamaged, medium. In contrast to seismic moment, the seismic potency (the spatial integral of slip) is an unambiguous quantity that has no discontinuity as $H$ approaches 0 . This consideration supports the idea that the seismic potency is a better parameter for the characterization of the overall size of a slip event [e.g., Heaton and Heaton, 1989; Ben-Zion, 1989].

\section{Can Vertically Stratified Bulk Structure Cause Shallow Coseismic Slip Deficit?}

[52] In this section, we further extend our analysis to the effects of near-surface low-rigidity bulk layers and study how these layers affect the depth dependence of slip in large earthquakes. We model earthquake sequences on a planar vertical strike-slip fault embedded into an elastic half-space (Figure 9a). The setup is similar to the depth-variable model of Lapusta et al. [2000], where friction acts in the top $24 \mathrm{~km}$ of the fault and the deeper extension moves with a prescribed plate rate of $35 \mathrm{~mm} /$ year.

[53] The physical parameters of the simulations presented in this work are shown in Figures 9a and 9b. The effective normal stress $\bar{\sigma}$ increases with depth and becomes uniform $(50 \mathrm{MPa})$ at depths greater than $2.6 \mathrm{~km}$ (Figure $9 \mathrm{~b}$ ). While we adopt this effective normal stress profile for convenience, as increasing normal stresses with depth would require adopting higher numerical resolution, such a profile is plausible for natural faults, due to fluid over-pressurization at depth. An early summary of evidence supporting fluid over-pressurization and a plausible mechanical model are presented by Rice [1992]. The constant value of normal stress with depth is consistent with the lack of systematic depth-dependence of stress drop for microseismicity, beyond that expected from the depth-dependency of crustal rigidity [e.g., Allmann and Shearer, 2007]. The variation of friction parameters $a$ and $b$ with depth shown in Figure 9b is similar to the one from Rice [1993] and Lapusta et al. [2000]; it is derived from laboratory experiments [Blanpied et al., 1995]. The region between $2.0 \mathrm{~km}$ and $14.3 \mathrm{~km}$ has velocity-weakening properties. The transition from velocity weakening to velocity strengthening at $14.3-\mathrm{km}$ depth is assumed to be associated with temperature increase with depth. The value of $L$ used is $8 \mathrm{~mm}$, in which case the model results in sequences of model-spanning earthquakes consistent with the results of Lapusta et al. [2000].

[54] We consider four different scenarios of earthquake sequences in: (1) homogeneous bulk structure without the shallow velocity-strengthening fault patch, (2) layered bulk structure without the shallow velocity-strengthening fault patch, (3) homogeneous bulk structure with the shallow velocity-strengthening fault patch, and (4) layered bulk structure with the shallow velocity-strengthening fault patch (Figure 9). The layered bulk model approximately corresponds to the 1-D Parkfield velocity structure down to the depth of $\sim 15 \mathrm{~km}$ used in the study by Custódio et al. [2005]. The scenarios with the velocity-strengthening patch at depths less than $2.0 \mathrm{~km}$ (Figure $9 \mathrm{~b}$ ) are motivated by laboratory experiments in which rock friction at low normal stress typically exhibits velocity-strengthening behavior due to unconsolidated fault gouge [e.g., Marone et al., 1991; Marone, 1998].

[55] Figures 10a and 10b show earthquake sequences simulated in the 2-D SEM model for scenarios 1 and 2. The solid lines are plotted every 5 years and show the continuous slow sliding (creep) of the velocity-strengthening region at depth. The slow slip creates a stress concentration at its tip and penetrates into the velocity-weakening region. In due time, an earthquake nucleates close to the transition. We show the progression of earthquakes with dashed lines plotted every second.

[56] The scenarios with homogeneous and layered bulk do not lead to shallow coseismic slip deficit in these particular 
(a)

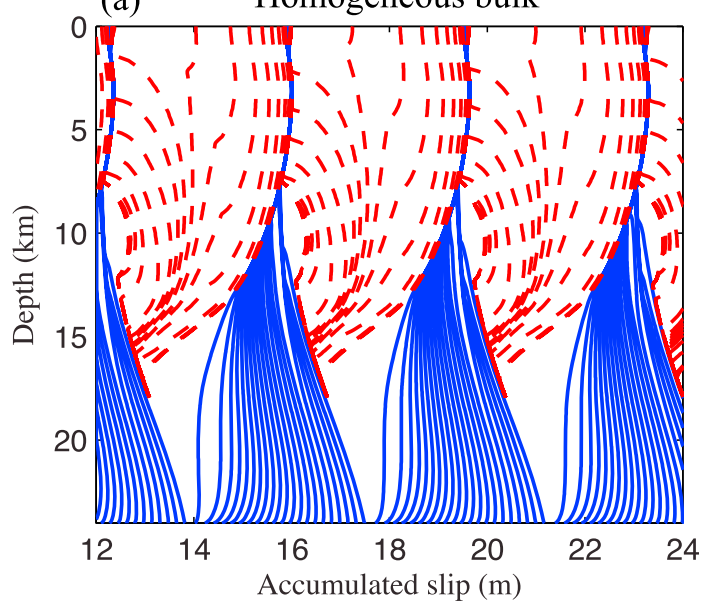

(c) Homogeneous bulk w/ VS patch

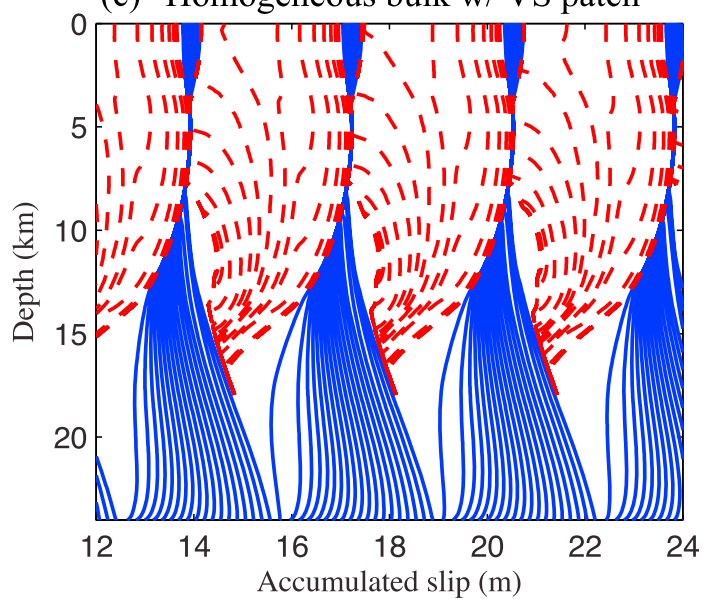

(e)

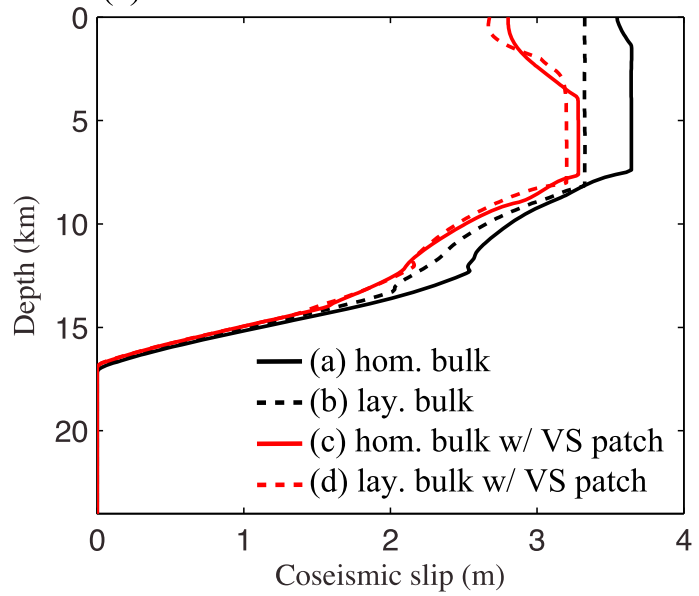

(b)

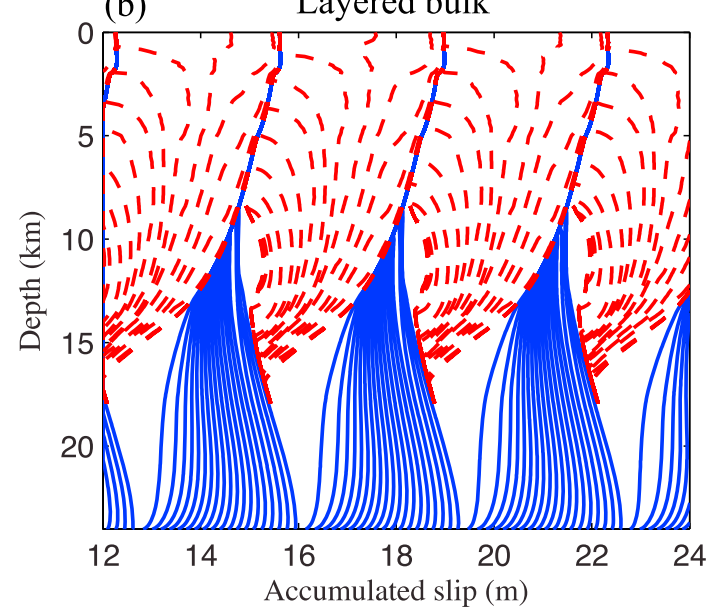

(d) Layered bulk w/ VS patch

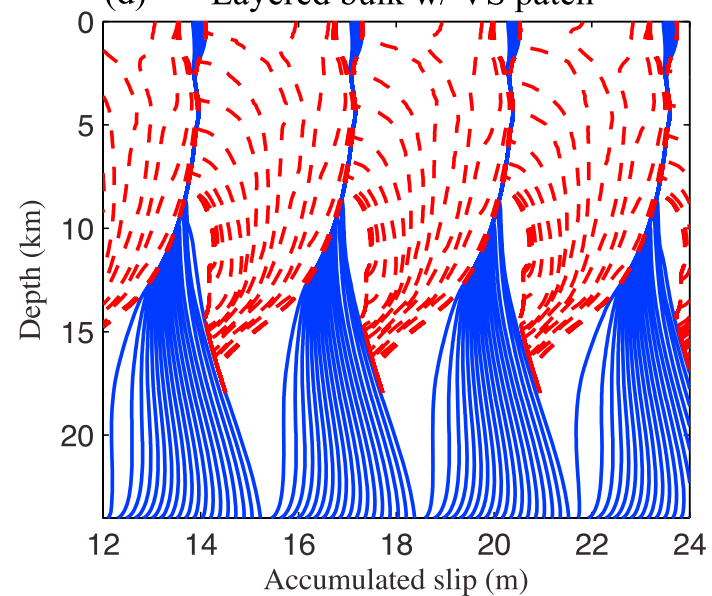

(f)

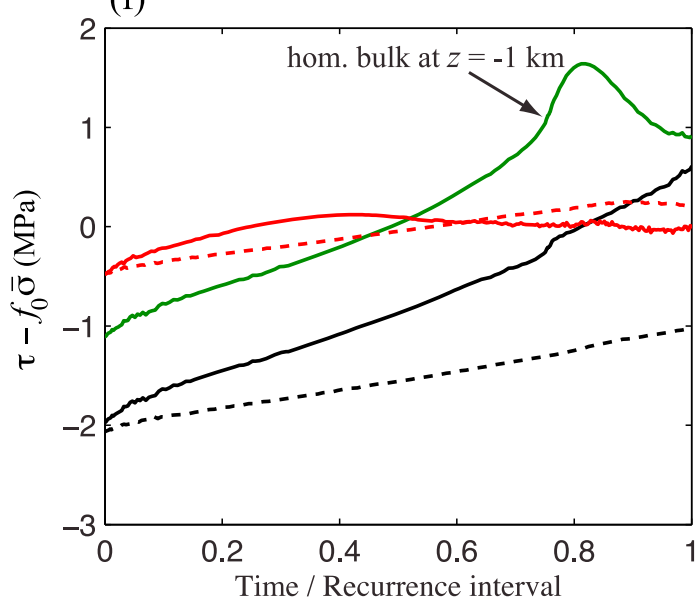

Figure 10. Simulated earthquake sequences and event characteristics of models with: (a) homogeneous bulk structure without the shallow velocity-strengthening fault patch, (b) layered bulk structure without the shallow velocity-strengthening fault patch, (c) homogeneous bulk structure with the shallow velocitystrengthening fault patch, and (d) layered bulk structure with the shallow velocity-strengthening fault patch. Solid lines show slip accumulation every 5 yrs. Dashed lines are intended to capture dynamic events and are plotted every $1 \mathrm{~s}$ during the earthquakes. (e) Seismic slip of a representative event in each case. Low-rigidity shallow bulk materials alone do not lead to coseismic slip deficit. (f) Shear stress evolution at the depth of $z=-2 \mathrm{~km}$ for all four cases and at the depth of $z=-1 \mathrm{~km}$ for the case 1 (Figure 10a). Time is normalized by the recurrence interval for each case, and $t=0$ corresponds to the time just after the prior seismic event. 
examples (Figures 10a, 10b, and 10e). From the evolution of shear stress in these cases (black and black dashed lines in Figure 10f), the stress accumulation rates in the lowerrigidity materials during the interseismic periods are smaller than those in the materials with higher rigidity. As a result, the prestress on the fault within the low-rigidity materials becomes smaller. However, as explained in section 4, the coseismic slip rates get amplified in the low-rigidity materials, resulting in the net effect on slip being nearly zero.

[57] In the case where the rigidity of the shallow bulk layers is low (Figure 10b), the rupture propagation is enhanced as the rupture propagates into the shallow layers. Although the absolute rupture speeds decelerate from $1.7 \mathrm{~km} / \mathrm{s}$ at $5-6 \mathrm{~km}$ depths to $1.2 \mathrm{~km} / \mathrm{s}$ at $1-2 \mathrm{~km}$ depths, the rupture speeds relative to the local shear wave speed $V_{\mathrm{r}} / V_{\mathrm{s}}$ increase from $V_{\mathrm{r}} / V_{\mathrm{s}}=$ 0.5 at $5-6 \mathrm{~km}$ depths to $V_{\mathrm{r}} / V_{\mathrm{s}}=0.7$ at $1-2 \mathrm{~km}$ depths. Hence despite the smaller prestress in the low-rigidity materials, the rupture accelerates with respect to the local $V_{\mathrm{s}}$ and the rupture propagation is enhanced, consistent with the dynamic amplification of the slip rates.

[58] The small reduction of the coseismic slip near the free surface in scenario 1 (the black curve in Figure 10e) is caused by minor aseismic creep. Due to this minor creep, interseismic shear stress near the free surface in scenario 1 decreases for some time (the green curve in Figure 10f). The occurrence of this minor creep is related to the decrease in effective normal stress $\bar{\sigma}$ toward the free surface. From the theoretical estimates of a nucleation size given in equations (15) and (16), $h^{*}$ is proportional to $\mu / \bar{\sigma}$. Since $\bar{\sigma}$ decreases next to the free surface, $h^{*}$ increases there, provided that other parameters are uniform over depth. The larger $h^{*}$ near the free surface in scenario 1 promotes aseismic slip there. This consideration also explains why scenario 2 is not similarly affected. Since rigidity $\mu$ is also smaller near the free surface in scenario $2, h^{*}$ remains relatively constant with depth, and hence the aseismic slip is not similarly promoted in that scenario.

[59] Figures $10 \mathrm{c}$ and $10 \mathrm{~d}$ show earthquake sequences simulated in the 2-D SEM model for scenarios 3 and 4 . The presence of the shallow velocity-strengthening patch leads to shallow coseismic slip deficit regardless of the properties of the bulk (red and red dashed lines in Figure 10e). As the rupture propagates over the velocity-strengthening area, it experiences additional resistance to slip. It still manages to propagate all the way to the surface, driven by the dynamic wave-mediated stress transfers, and to accumulate quite a bit of slip. However, the slip is lower than at depth. The gradient in slip keeps the stresses in the shallow part sufficiently high to allow for some afterslip and interseismic creep. Due to the interseismic creep and afterslip in the shallow velocity-strengthening region, the stress accumulation rates there during the interseismic periods are small in both scenarios (red and red dashed lines in Figure 10f).

[60] The results here suggest that coseismic slip deficit can be caused by the presence of a shallow velocitystrengthening region, but not by that of low-rigidity shallow bulk materials. In a model embedded in elastic media, the accumulated slip is equal to the sum of co-, inter- and postseismic slip. On velocity-weakening faults accommodating little inter- and postseismic slip, the coseismic slip at a given point has to catch up with that on the rest of the fault plane. Hence the cancelation of the net effect of dynamic amplification and low interseismic stress accumulation in
Figure 10 is reasonable. The studies by Rybicki [1992] and Rybicki and Yamashita [1998], which proposed that the reduction of coseismic slip at shallow depths can be caused by the presence of low-rigidity materials, did not consider dynamic amplification of coseismic slip, even though they considered a wider range of conditions. While exploring the wider range of parameters may be important, our conclusions should still be valid, unless certain conditions lead to significant interseismic creep or afterslip on faults with velocity-weakening friction.

\section{Conclusions}

[61] We have developed a 2-D SEM algorithm for simulating long-term histories of seismic and aseismic fault slip on a vertical strike-slip fault embedded in heterogeneous bulk media subjected to slow tectonic loading. Our approach reproduces all stages of earthquake cycles from accelerating slip before dynamic instability, to rapid dynamic propagation of earthquake rupture, to postseismic slip, and to interseismic creep. We have set up an antiplane benchmark problem and have verified the developed SEM approach by comparing SEM and BIM simulation results in a 2-D model of small repeating earthquakes.

[62] Using the developed formulation, we have investigated the effects of variable fault zone bulk properties on source properties of small repeating earthquakes. Our results suggest that source properties of small repeating earthquakes depend on the width of a lower-rigidity bulk (or a damaged zone) and its rigidity value. We find that a fault bisecting a lower-rigidity layer, compared to the one in undamaged country rock, leads to the following changes in the properties of earthquakes and their cycles: (1) reduction in the earthquake nucleation size, (2) amplification of slip rates during dynamic rupture propagation, (3) increase in the recurrence interval, and (4) smaller amount of aseismic slip. Note that changes 2-4 are due to a combined effect of the presence of the lower-rigidity layer and of the change 1.

[63] In this work, a damaged fault zone is characterized by the region of a low-rigidity linear elastic medium. However, the actual damage zones may differ from the undamaged rock in other important ways, e.g. due to spontaneous generation and healing of physical damage and the associated evolution of elastic moduli. Such additional features may further affect the source properties of small repeating earthquakes. The presented SEM framework can be extended to include offfault inelastic deformation [e.g., Andrews, 2005; Templeton and Rice, 2008] and spontaneous damage evolution in the context of a continuum damage mechanics formulation [e.g., Lyakhovsky et al., 1997], which can be used to explore and quantify such effect.

[64] We have further examined the effects of vertically stratified bulk layers on the nature of shallow coseismic slip deficit. For the set of parameters we have considered, lowrigidity shallow bulk materials alone do not lead to coseismic slip deficit. While the low-rigidity materials do cause lower interseismic stress accumulation, they also cause dynamic amplification of coseismic slip rates, with the net effect on slip being nearly zero. At the same time, the addition of velocity-strengthening friction to shallow parts of the fault leads to coseismic slip deficit in all cases we have considered. 
[65] While velocity-strengthening friction leads to the reduction of coseismic slip near the Earth surface, there remains a question of how the coseismic slip deficit is accommodated throughout the earthquake cycle. The consequence of velocitystrengthening fault friction at shallow depths is that the deficit of coseismic slip is relieved by postseismic afterslip and interseismic creep. However, several seismic events with inferred shallow slip deficit discussed in section 1 are not associated with either resolvable shallow interseismic creep or robust shallow afterslip [Jacobs et al., 2002; Fialko, 2004; Fialko et al., 2005; Fielding et al., 2009]. In such cases, the slip deficit in the shallow parts of the fault may be due to either smaller shallow seismic events or distributed inelastic bulk deformation. Understanding the typical origin of shallow slip deficit remains a subject of future work.

[66] The developed SEM methodology can be used to study a number of fault slip phenomena that bridge the broad spectrum of fault slip behavior, from rupture dynamics to long-term crustal deformation. The SEM model allows for more flexibility in fault geometry and heterogeneous and non-elastic bulk properties in long-term simulations of fault slip. Furthermore, while the methodology is presented using the 2-D antiplane problem, it can be extended to the 2-D in-plane and 3-D formulations if efficient implicit solvers for 3-D problems are constructed. The developed models would be useful for interpreting seismic and geological data collected and sampled close to structurally complex fault zones at SAFOD and other drilling sites.

\section{Appendix A: Fault Boundary Matrix}

[67] The fault surface $\Gamma$ consists of quadrilateral elements $\Gamma_{e}$ inherited from hexahedral elements lying on the two sides $\Gamma_{ \pm}$of the fault. The matrix $\mathbf{B}$ in (2) is a sparse rectangular matrix obtained by assembling the contributions $\mathbf{B}_{e}$ from each of the fault boundary elements $\Gamma_{e}$ that are the same for the three components of traction. The term of $\mathbf{B}_{e}$ associated with the GLL node with local indices $(i, j)$ in $\Gamma_{e} \in \Gamma_{ \pm}$is

$$
B_{i j, i j}^{e \pm}= \pm \omega_{i} \omega_{j} J_{e}^{i j},
$$

where $\omega_{k}$ denote the weights associated with the GLL integration quadrature and

$$
J_{e}^{i j}=\left\|\frac{\partial \mathbf{x}}{\partial \xi} \times \frac{\partial \mathbf{x}}{\partial \eta}\right\|\left(\xi_{i}, \eta_{j}\right)
$$

is the Jacobian of the coordinate transformation from $\mathbf{x} \in \Gamma_{e}$ to $\boldsymbol{\xi}=(\xi, \eta) \in[-1,1]^{2}$.

[68] The outward normal vector of the fault boundary $\Gamma^{+}$ is obtained by

$$
\mathbf{n}(\xi, \eta)=\frac{1}{J_{e}} \frac{\partial \mathbf{x}}{\partial \xi} \times \frac{\partial \mathbf{x}}{\partial \eta} .
$$

\section{Appendix B: Quasi-Static and Dynamic Time Stepping Algorithms Used in This Study}

[69] The algorithm for the dynamic scheme was discussed by Kaneko et al. [2008] in more detail. Note that the notations used in the dynamic scheme is slightly different from those of Kaneko et al. [2008].
[70] Quasi-static scheme

1. Predict displacement on the fault:

$$
\mathbf{u}^{\mathrm{f} *}=\mathbf{u}^{\mathrm{f}}+\Delta t \dot{\mathbf{u}}^{\mathrm{f}}
$$

2. Solve for displacement in the medium:

$$
\mathbf{K}_{22} \mathbf{u}^{\mathrm{m} *}=-\mathbf{K}_{21} \mathbf{u}^{\mathrm{f} *}
$$

3. Compute $\mathbf{f}$ on the fault:

$$
\mathbf{f}^{*} \leftarrow \mathbf{K}_{11} \mathbf{u}^{\mathrm{f} *}+\mathbf{K}_{12} \mathbf{u}^{\mathrm{m} *}
$$

4. Compute traction on the fault:

$$
\boldsymbol{\tau}^{\mathrm{tot} *}=\boldsymbol{\tau}_{\mathrm{o}}-\left(\mathbf{B}_{+}+\mathbf{B}_{-}\right)^{-1}\left(\mathbf{f}_{+}^{*}-\mathbf{f}_{-}^{*}\right)
$$

5. Compute $\dot{\delta}^{*}$ from a friction law together with $\tau^{\text {tot* }}$

6. Correct displacement on the fault:

$$
\mathbf{u}^{\mathrm{f} * *}=\mathbf{u}^{\mathrm{f}}+\frac{\Delta t}{2}\left(\dot{\mathbf{u}}^{\mathrm{f}}+\dot{\mathbf{u}}^{\mathrm{f} *}\right)
$$

7. Repeat 2-5 to obtain $\mathbf{u}^{\mathrm{m} * *}, \tau^{\mathrm{tot} * *}$, and $\dot{\delta}^{* *}$

[71] Dynamic scheme

1. Update displacement and partial velocity:

$$
\begin{gathered}
\mathbf{u} \leftarrow \mathbf{u}+\Delta t \dot{\mathbf{u}}+\frac{\Delta t^{2}}{2} \ddot{\mathbf{u}} \\
\dot{\mathbf{u}}^{*}=\dot{\mathbf{u}}+\frac{\Delta t^{2}}{2} \ddot{\mathbf{u}}
\end{gathered}
$$

2. Compute the internal forces:

$$
\mathbf{f}^{\mathrm{i}} \leftarrow-\mathbf{K u}
$$

3. Compute the 'stick' traction (equation [18] of Kaneko et al. [2008]):

$$
\begin{gathered}
\dot{\mathbf{u}}^{\text {free }}=\dot{\mathbf{u}}^{*}-\frac{\Delta t}{2}\left(\mathbf{M}^{-1} \mathbf{f}^{\mathbf{i}}\right) \\
\tilde{\boldsymbol{\tau}}^{\text {tot }}=\tau_{o}+\mathbf{Z} \dot{\delta}^{\text {free }}
\end{gathered}
$$

4. Find fault traction and slip velocity satisfying a friction law and the relation

$$
\tau^{\mathrm{tot}}=\tilde{\tau}^{\mathrm{tot}}-\mathbf{Z} \dot{\delta}
$$

5. Add the fault boundary term to the sum of internal forces:

$$
\mathbf{f}^{\mathrm{i}} \leftarrow \mathbf{f}^{\mathrm{i}}+\mathbf{B} \boldsymbol{\tau}
$$

6. Solve for acceleration:

$$
\ddot{\mathbf{u}} \leftarrow \mathbf{M}^{-1} \mathbf{f}^{\mathrm{i}}
$$

7. Complete the update of velocity:

$$
\dot{\mathbf{u}} \leftarrow \dot{\mathbf{u}}^{*}+\frac{\Delta t}{2} \ddot{\mathbf{u}}
$$

\section{Appendix C: Variable Evolution Time Step in 2-D Antiplane Problems}

[72] Simulations of long-term deformation histories with periods of rapid dynamic slip (earthquakes) require time steps that change by orders of magnitude. For the SEM with 


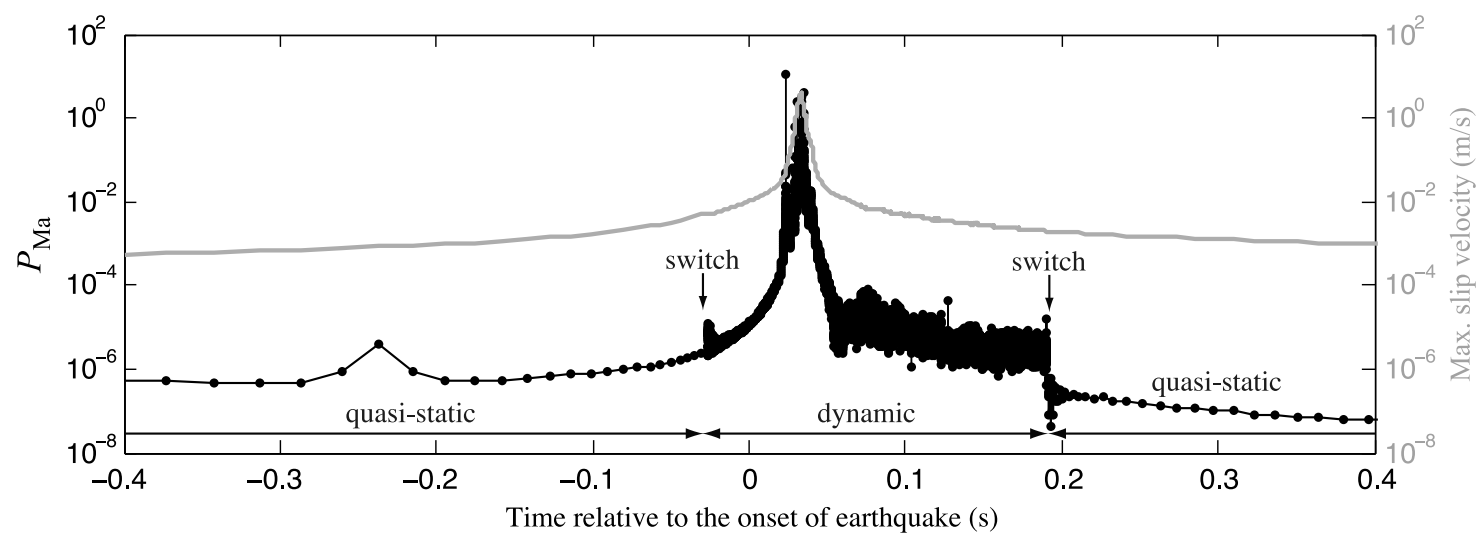

Figure D1. The ratio $P_{\mathrm{Ma}}$ of the inertial term to the other terms in the governing equation and the maximum slip velocity on the fault as a function of time before, during and after the simulated earthquake shown in Figure 4. $P_{\mathrm{Ma}}$ is defined in equations (D1) and (D2). At the times of the switch from quasistatic to dynamic and vice versa, the inertial term Mü is much smaller $\left(\sim 10^{-6}\right)$ than the other terms in the governing equation (2).

the implicit scheme, we adopt the time stepping scheme developed for BIM by Lapusta et al. [2000] for a 2-D antiplane problem. This scheme also works well for our SEM model. Note that the maximum time step is limited by the Courant condition and constant in the SEM with the explicit scheme, but not in the implicit scheme (unconditionally stable). The variable time step $\Delta t$ is chosen as:

$$
\Delta t=\max \left\{\Delta t_{\min }, \Delta t_{\mathrm{ev}}\right\}
$$

where $\Delta t_{\text {min }}$ is the minimum time step, and $\Delta t_{e v}$ depends on slip velocity at each time step. The minimum time step is set by the Courant condition and given by

$$
\Delta t_{\min }=C \Delta x_{\min } / V_{\mathrm{s}},
$$

where $C=0.6$ is used in our 2-D antiplane problem. The same condition is used for modeling single dynamic ruptures in the 2-D antiplane test problem of Kaneko et al. [2008]. The time step $\Delta t_{\mathrm{ev}}$ is set to be inversely proportional to slip velocity:

$$
\Delta t_{\mathrm{ev}}=\min \left[\xi_{i} L_{i} / \dot{\delta}_{i}\right]
$$

where $L_{i}, \dot{\delta}_{i}$, and $\xi_{i}$ are the characteristic slip, the current slip velocity and a prescribed parameter for the $i$ th fault node of the discretized domain, respectively. $\xi_{i}$ is a function of friction properties from linear stability analysis [Lapusta et al., 2000], and it is constrained to satisfy $\xi_{i} \leq \xi_{c}$, where $\xi_{c}$ is a constant, to ensure that slip at each time step does not exceed $\xi_{c} L_{i}$. As in the work of Lapusta et al. [2000], we use $\xi_{c}=1 / 2$ in our 2-D SEM and BIM models.

\section{Appendix D: Criteria for Switching Between Quasi-Static and Fully Dynamic SEMs}

[73] The quasi-static SEM formulated in section 2 can be merged with the fully dynamic SEM [Kaneko et al., 2008]. We switch from the quasi-static to the dynamic SEM and vice versa based on the values of the maximum slip velocity on the fault described in section 3.1. To make sure that at the times of the switch the inertial term is negligible relative to the other terms in the governing equation, we define and compute the ratio $P_{\mathrm{Ma}}$ of the inertial term on the fault to the other terms at each time step:

$$
P_{\mathrm{Ma}}=\max \left(\max \left[\frac{\left|\mathbf{M} \ddot{\mathbf{u}}^{\mathrm{eff}}\right|}{|\mathbf{K u}|}\right], \max \left[\frac{\left|\mathbf{M} \ddot{\mathbf{u}}^{\mathrm{eff}}\right|}{|\mathbf{B} \boldsymbol{\tau}|}\right]\right)
$$

during the quasi-static regime, and

$$
P_{\mathrm{Ma}}=\max \left(\max \left[\frac{|\mathbf{M} \ddot{\mathbf{u}}|}{|\mathbf{K u}|}\right], \max \left[\frac{|\mathbf{M} \ddot{\mathbf{u}}|}{|\mathbf{B} \boldsymbol{\tau}|}\right]\right)
$$

during the dynamic regime. The effective acceleration computed during the quasi-static regime is computed as $\ddot{\mathbf{u}}^{\text {eff }} \equiv\left(\dot{\mathbf{u}}_{n+1}-\dot{\mathbf{u}}_{n}\right) / \Delta t$. Figure D1 shows $P_{\mathrm{Ma}}$ and values of the maximum slip velocity on the fault as a function of time before, during and after one of the simulated earthquake events shown in Figure 4. At the times of the switch, the inertial term Mü is negligible relative to the other terms in the governing equation, verifying the use of our switching criteria based on the values of the maximum slip velocity.

\section{Appendix E: Nucleation Size for a Layered Elastic Medium}

[74] We derive the theoretical estimate of a nucleation size on rate and state faults embedded in a simple layered medium shown in Figure 2 . The static stiffness $k_{\text {hom }}$ for sliding on a patch of characteristic size $h$ in a homogeneous elastic solid is given by [e.g., Dieterich 1992]:

$$
k_{\mathrm{hom}}=\frac{\gamma \mu}{h}
$$

where $\gamma$ is a parameter of order one that depends on the geometry of the slip patch and assumptions relating to slip or stress conditions on the patch, and $\mu$ is the rigidity. Considering sinusoidal perturbations of slip of wavelength $h$, for which $\gamma=\pi$, Rice and Ruina [1983] and Ruina [1983] showed that frictional sliding with the rate and state friction 
is always stable for the perturbations of the shortest wavelengths, but, if the sliding surfaces have velocity-weakening properties, then a critical wavelength exists, associated to a critical stiffness $k_{\mathrm{c}}$, such that larger wavelengths are unstable. This means that only the long wavelengths of a perturbation, with $k_{\mathrm{hom}}<k_{\mathrm{c}}$, diverge exponentially and trigger an instability. For rate and state friction with the aging law (11), the critical stiffness is $k_{\mathrm{c}}=\bar{\sigma}(b-a) / L[$ Ruina, 1983]. Setting $k_{r m c}=k_{\text {hom }}$ and solving for the critical size $h$ leads to equation (15), which we denote $h_{\text {hom }}^{*}$ in this appendix.

[75] Ampuero et al. [2002] derived an analytical expression of the static stiffness $k_{\text {lay }}$ for sinusoidal slip perturbations of wavelength $h$ in a simple layered medium. We slightly modify their expression by introducing a parameter $\gamma$ that accommodates more general slip modes, inspired by equation (E1):

$$
k_{\text {lay }}=\frac{\gamma \mu_{\mathrm{D}}}{h} \operatorname{cotanh}\left[2 H \frac{\gamma}{h}+\operatorname{arctanh}\left(\frac{\mu_{\mathrm{D}}}{\mu}\right)\right],
$$

where $\mu_{\mathrm{D}}$ and $\mu$ are rigidity of the low-rigidity layer adjacent to the fault plane and that of the undamaged host rock, respectively, and $H$ is the thickness of the low-rigidity layer (Figure 2). Since $k_{\mathrm{c}}$ depends only on the friction law and effective normal stress $\bar{\sigma}, k_{\mathrm{c}}$ is the same for both the homogeneous and layered cases. By setting $k_{\text {hom }}=k_{\text {lay }}=k_{\mathrm{c}}$, we obtain

$$
h_{\text {lay }}^{*} \tanh \left[2 H \frac{\gamma}{h_{\text {lay }}^{*}}+\operatorname{arctanh}\left(\frac{\mu_{\mathrm{D}}}{\mu}\right)\right]=h_{\text {hom }}^{*} .
$$

Given $h_{\text {hom }}^{*}$, one can find $h_{\text {lay }}^{*}$ by numerically solving equation (E3). The parameter $\gamma$ can be determined empirically for a given model geometry. For the antiplane problems we consider here, we find that setting $\gamma=\pi / 4$ provides a satisfactory representation of our simulation results.

[76] Acknowledgments. This study was supported by the National Science Foundation (grants EAR-0548277 and EAR-0944288), the Southern California Earthquake Center (SCEC), and the Gordon and Betty Moore Foundation. SCEC is funded by NSF cooperative agreement EAR-0106924 and USGS cooperative agreement 02HQAG0008. The SCEC contribution number for this paper is 1477 . This is Caltech Tectonics Observatory contribution 161. We thank Eun-Seo Choi, Billy Landuyt, Ittai Kurzon, and Chris Davies for general discussion on numerical methods. The reviews by Brad Aagaard and an anonymous reviewer helped us improve the manuscript.

\section{References}

Allmann, B. B., and P. M. Shearer (2007), Spatial and temporal stress drop variations in small earthquakes near Parkfield, California, J. Geophys. Res., 112, B04305, doi:10.1029/2006JB004395.

Ampuero, J.-P. (2002), Etude physique et numérique de la nucléation des séismes, Ph.D. thesis, Univ. Paris 7, Denis Diderot, Paris.

Ampuero, J.-P., J.-P. Vilotte, and F. J. Sanchez-Sesma (2002), Nucleation of rupture under slip dependent friction law: Simple models of fault zone, J. Geophys. Res., 107(B12), 2324, doi:10.1029/2001JB000452.

Andrews, D. J. (2005), Rupture dynamics with energy loss outside the slip zone, J. Geophys. Res., 110, B01307, doi:10.1029/2004JB003191.

Ben-Zion, Y. (1989), The response of two joined quarter spaces to $S H$ line sources located at the material discontinuity interface, Geophys. J. Int., 98(2), 213-222, doi:10.1111/j.1365-246X.1989.tb03346.x.

Bilham, R. (2010), Lessons from the Haiti earthquake, Nature, 463, 878-879, doi:10.1038/463878a.
Blanpied, M. L., D. A. Lockner, and J. D. Byerlee (1995), Frictional slip of granite at hydrothermal conditions, J. Geophys. Res., 100, 13,045-13,064, doi:10.1029/95JB00862.

Blanpied, M. L., C. J. Marone, and D. A. Lockner (1998), Quantitative measure of the variation in fault rheology due to fluid-rock interactions, J. Geophys. Res., 103, 9691-9712, doi:10.1029/98JB00162.

Chen, K. H., R. Bürgmann, R. M. Nadeau, T. Chen, and N. Lapusta (2010), Postseismic variations in seismic moment and recurrence interval of repeating earthquakes, Earth Planet. Sci. Lett., 299, 118-125, doi:10.1016/j. eps1.2010.1008.1027.

Chen, T., and N. Lapusta (2009), Scaling of small repeating earthquakes explained by interaction of seismic and aseismic slip in a rate and state fault model, J. Geophys. Res., 114, B01311, doi:10.1029/2008JB005749.

Chester, F. M., J. P. Evans, and R. L. Biegel (1993), Internal structure and weakening mechanisms of the San Andreas Fault, J. Geophys. Res., 98, 771-786, doi:10.1029/92JB01866.

Cochard, A., and R. Madariaga (1996), Complexity of seismicity due to highly rate-dependent friction, J. Geophys. Res., 101, 25,321-25,336, doi:10.1029/96JB02095.

Custódio, S., P. Liu, and R. J. Archuleta (2005), The $2004 \mathrm{M}_{\mathrm{w}} 6.0$ Parkfield, California, earthquake: Inversion of near-source ground motion using multiple data sets, Geophys. Res. Lett., 32, L23312, doi:10.1029/ 2005 GL024417.

Day, S. M., L. A. Dalguer, N. Lapusta, and Y. Liu (2005), Comparison of finite difference and boundary integral solutions to three-dimensional spontaneous rupture, J. Geophys. Res., 110, B12307, doi:10.1029/ 2005JB003813.

Dieterich, J. H. (1978), Time-dependent friction and the mechanics of stick-slip, Pure Appl. Geophys., 116, 790-806, doi:10.1007/BF00876539.

Dieterich, J. H. (1979), Modeling of rock friction: 1. Experimental results and constitutive equations, J. Geophys. Res., 84, 2161-2168, doi:10.1029/ JB084iB05p02161.

Dieterich, J. H. (1992), Earthquake nucleation on faults with rate- and state-dependent strength, Tectonophysics, 211, 115-134, doi:10.1016/ 0040-1951(92)90055-B.

Dieterich, J. H., and B. H. Kilgore (1994), Direct observation of frictional contacts: New insights for state-dependent properties, Pure Appl. Geophys., 143, 283-302, doi:10.1007/BF00874332.

Dieterich, J. H., and K. B. Richards-Dinger (2010), Earthquake recurrence in simulated fault systems, Pure Appl. Geophys., 167, 1087-1104, doi:10.1007/s00024-010-0094-0.

Duan, B., and D. D. Oglesby (2005), The dynamics of thrust and normal faults over multiple earthquake cycles: Effects of dipping fault geometry, Bull. Seismol. Soc. Am., 95(5), 1623-1636, doi:10.1785/0120040234.

Dupros, F., F. D. Martin, E. Foerster, D. Komatitsch, and J. Roman (2010), High-performance finite-element simulations of seismic wave propagation in three-dimensional nonlinear inelastic geological media, Parallel Comput., 36, 308-325, doi:10.1016/j.parco.2009.12.011.

Fialko, Y. (2004), Evidence of fluid-filled upper crust from observations of post-seismic deformation due to the $1992 M_{w} 7.3$ Landers earthquake, J. Geophys. Res., 109, B08401, doi:10.1029/2004JB002985.

Fialko, Y., D. Sandwell, M. Simons, and P. Rosen (2005), Three-dimensional deformation caused by the Bam, Iran, earthquake and the origin of shallow slip deficit, Nature, 435, 295-299, doi:10.1038/nature03425.

Fielding, E. J., P. R. Lundgren, R. Bürgmann, and G. J. Funning (2009), Shallow fault-zone dilatancy recovery after the 2003 bam earthquake in Iran, Nature, 458, 64-68, doi:10.1038/nature07817.

Harris, R. A., and S. M. Day (1997), Effects of a low-velocity zone on a dynamic rupture, Bull. Seismol. Soc. Am., 87, 1267-1280.

Heaton, T. H., and R. E. Heaton (1989), Static deformations from point forces and force couples located in welded elastic Poissonian half-spaces: Implications for seismic moment tensors, Bull. Seismol. Soc. Am., 79, 813-841.

Hestenes, M. R., and E. Stiefel (1952), Methods of conjugate gradients for solving linear systems, J. Res. Natl. Bur. Stand., 49, 409-436.

Hillers, G., Y. Ben-Zion, and P. M. Mai (2006), Seismicity on a fault controlled by rate- and state-dependent friction with spatial variations of the critical slip distance, J. Geophys. Res., 111, B01403, doi:10.1029/ 2005JB003859.

Ida, Y. (1973), The maximum acceleration of seismic ground motion, Bull. Seismol. Soc. Am., 63, 959-968.

Jacobs, A., D. Sandwell, Y. Fialko, and L. Sichoix (2002), The 1999 $\left(M_{w}\right.$ 7.1) Hector Mine, California, earthquake: Near-field postseismic deformation from ERS interferometry, Bull. Seismol. Soc. Am., 92, 1433-1442, doi:10.1785/0120000908.

Kaneko, Y., and N. Lapusta (2008), Variability of earthquake nucleation in continuum models of rate-and-state faults and implications for aftershock rates, J. Geophys. Res., 113, B12312, doi:10.1029/2007JB005154. 
Kaneko, Y., and N. Lapusta (2010), Supershear transition due to a free surface in 3-D simulations of spontaneous dynamic rupture on vertical strike-slip faults, Tectonophysics, 493, 272-284, doi:10.1016/j. tecto.2010.06.015.

Kaneko, Y., N. Lapusta, and J.-P. Ampuero (2008), Spectral element modeling of spontaneous earthquake rupture on rate and state faults: Effect of velocity-strengthening friction at shallow depths, J. Geophys. Res., 113 , B09317, doi:10.1029/2007JB005553.

Kato, N. (2004), Interaction of slip on asperities: Numerical simulation of seismic cycles on a two-dimensional planar fault with nonuniform frictional property, J. Geophys. Res., 109, B12306, doi:10.1029/2004JB003001.

Komatitsch, D., and J. Tromp (1999), Introduction to the spectral element method for three-dimensional seismic wave propagation, Geophys. J. Int., 139, 806-822, doi:10.1046/j.1365-246x.1999.00967.x.

Komatitsch, D., and J.-P. Vilotte (1998), The spectral element method: An efficient tool to simulate the seismic response of 2D and 3D geological structures, Bull. Seismol. Soc. Am., 88, 368-392.

Lapusta, N., and Y. Liu (2009), Three-dimensional boundary integral modeling of spontaneous earthquake sequences and aseismic slip, J. Geophys. Res., 114, B09303, doi:10.1029/2008JB005934.

Lapusta, N., J. Rice, Y. Ben-Zion, and G. Zheng (2000), Elastodynamic analysis for slow tectonic loading with spontaneous rupture episodes on faults with rate- and state-dependent friction, J. Geophys. Res., 105, 23,765-23,789, doi:10.1029/2000JB900250.

Liu, Y., and J. R. Rice (2005), Aseismic slip transients emerge spontaneously in 3D rate and state modeling of subduction earthquake sequences, J. Geophys. Res., 110, B08307, doi:10.1029/2004JB003424.

Lottes, J. W., and P. F. Fischer (2004), Hybrid multigrid/Schwarz algorithms for the Spectral Element Method, J. Sci. Comp., 24, 45-78, doi:10.1007/s10915-004-4787-3.

Lyakhovsky, V. Y., Y. Ben-Zion, and A. Agnon (1997), Distributed damage, faulting, and friction, J. Geophys. Res., 102, 27,635-27,649, doi:10.1029/ 97JB01896.

Marone, C. (1998), Laboratory-derived friction laws and their application to seismic faulting, Annu. Rev. Earth Planet. Sci., 26, 643-696, doi:10.1146/annurev.earth.26.1.643.

Marone, C., C. H. Scholz, and R. Bilham (1991), On the mechanics of earthquake afterslip, J. Geophys. Res., 96, 8441-8452, doi:10.1029 91JB00275.

Perfettini, H., and J.-P. Ampuero (2008), Dynamics of a velocity strengthening region: Implications for slow earthquakes and postseismic slip, J. Geophys. Res., 113, B09411, doi:10.1029/2007JB005398.

Rice, J. R. (1992), Fault stress states, pore pressure distribution, and the weakness of the San Andreas Fault, in Fault Mechanics and Transport Properties of Rocks, edited by B. Evans and T. Wong, pp. 475-503, Academic, San Diego, Calif., doi:10.1016/S0074-6142(08)62835-1.

Rice, J. R. (1993), Spatio-temporal complexity of slip on a fault, J. Geophys. Res., 98, 9885-9907, doi:10.1029/93JB00191.

Rice, J. R., and Y. Ben-Zion (1996), Slip complexity in earthquake fault models, Proc. Natl. Acad. Sci. U. S. A., 93, 3811-3818, doi:10.1073/ pnas.93.9.3811.
Rice, J. R., and A. L. Ruina (1983), Stability of steady frictional slipping, J. Appl. Mech., 50, 343-349, doi:10.1115/1.3167042.

Rubin, A. M., and J.-P. Ampuero (2005), Earthquake nucleation on (aging) rate and state faults, J. Geophys. Res., 110, B11312, doi:10.1029/ 2005JB003686.

Ruina, A. L. (1983), Slip instability and state variable friction laws, J. Geophys. Res., 88, 10,359-10,370, doi:10.1029/JB088iB12p10359.

Rundle, J. B., W. Klein, and S. Gross (1999), Physical basis for statistical patterns in complex earthquake populations: Models, predictions and tests, Pure Appl. Geophys., 155, 575-607, doi:10.1007/s000240050278.

Rybicki, K. R. (1992), Strike-slip faulting in the presence of low-rigidity inhomogeneities, Bull. Seismol. Soc. Am., 82, 2170-2190.

Rybicki, K. R., and T. Yamashita (1998), Faulting in vertically inhomogeneous media and its geophysical implications, Geophys. Res. Lett., 25, 2893-2896, doi:10.1029/2002GL014672.

Shibazaki, B., and M. Matsu'ura (1992), Spontaneous processes for nucleation, dynamic propagation, and stop of earthquake rupture, Geophys. Res. Lett., 19, 1189-1192, doi:10.1029/92GL01072.

Simons, M., Y. Fialko, and L. Rivera (2002), Coseismic deformation from the $1999 \mathrm{Mw} 7.1$ Hector Mine, California, earthquake as inferred from InSAR and GPS observations, Bull. Seismol. Soc. Am., 92, 1390-1402, doi:10.1785/0120000933.

Templeton, E. L., and J. R. Rice (2008), Off-fault plasticity and earthquake rupture dynamics: 1. Dry materials or neglect of fluid pressure changes, J. Geophys. Res., 113, B09306, doi:10.1029/2007JB005529.

Trefethen, L. N., and D. Bau (1997), Numerical Linear Algebra, Soc. for Indust. and Appl. Math., Philadelphia, Pa.

Tullis, T. E. (1996), Rock friction and its implications for earthquake prediction examined via models of Parkfield earthquakes, Proc. Natl. Acad. Sci. U. S. A., 93, 3803-3810.

Ward, S. (1997), Dogtails versus rainbows: Synthetic earthquake rupture models as an aid in interpreting geological data, Bull. Seismol. Soc. Am., 87, 1422-1441.

Zampieri, E., and L. F. Pavarino (2006), Implicit spectral element methods and Neumann-Neumann preconditioners for acoustic waves, Comput. Methods Appl. Mech. Eng., 195, 2649-2673, doi:10.1016/j.cma.2005.06.005.

Zoback, M., S. Hickman, and W. Ellsworth (2010), Scientific drilling into the San Andreas Fault zone, Eos Trans. $A G U, 91(22), 197$, doi:10.1029/ 2010EO220001

J.-P. Ampuero, Division of Geological and Planetary Sciences, California Institute of Technology, 1200 E. California Blvd., MC 252-21, Pasadena, CA 91125, USA. (ampuero@gps.caltech.edu)

Y. Kaneko, Institute of Geophysics and Planetary Physics, Scripps Institution of Oceanography, Univerisity of California, San Diego, 9500 Gilman Dr., MC 0225, La Jolla, CA 92093, USA. (ykaneko@ucsd.edu)

N. Lapusta, Division of Engineering and Applied Science, California Institute of Technology, 1200 E. California Blvd., MC 104-44, Pasadena, CA 91125, USA. (lapusta@caltech.edu) 